\title{
Wireless surface acoustic wave sensors for displacement and crack monitoring in concrete structures
}

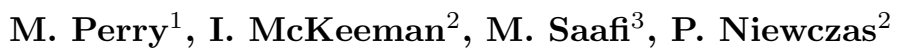 \\ 1 Department of Civil and Environmental Engineering, James Weir Building, \\ University of Strathclyde, Glasgow, G1 1XJ \\ 2 Department of Electronic and Electrical Engineering, Royal College Building, \\ University of Strathclyde, Glasgow, G1 1XW \\ 3 Department of Engineering, Lancaster University, Lancaster, LA1 4YR \\ E-mail: ${ }^{1} \mathrm{~m}$.perry@strath.ac.uk, ${ }^{3} \mathrm{~m}$.saafi@lancaster.ac.uk
}

\begin{abstract}
.
In this work, we demonstrate that wireless surface acoustic wave devices can be used to monitor millimetre displacements in crack opening during the cyclic and static loading of reinforced concrete structures. Sensors were packaged to extend their gauge length and to protect them against brittle fracture, before being surface-mounted onto the tensioned surface of a concrete beam. The accuracy of measurements was verified using computational methods and optical-fibre strain sensors. After packaging, the displacement and temperature resolutions of the surface acoustic wave sensors were $10 \mu \mathrm{m}$ and $2{ }^{\circ} \mathrm{C}$ respectively. With some further work, these devices could be retrofitted to existing concrete structures to facilitate wireless structural health monitoring.
\end{abstract}




\section{Introduction}

Rapid developments in wireless sensing have been driven by a desire for compact devices which allow for flexible and rapid installation [1]. Wireless sensor networks have already been successfully deployed in various structural health monitoring (SHM) applications $[2,3,4]$, but the limited battery life of wireless sensors typically leads to reduced performance levels, or increased costs from the development of data compression or energy harvesting methods $[5,6]$.

Surface acoustic wave (SAW) devices are a class of wireless sensor which require no batteries, as they operate on wireless power. By utilising radio-frequency identification (RFID) technology, SAW sensors can provide passive measurements of strain, temperature, pressure, acceleration and chemical contamination, even in harsh environments $[7,8,9,10,11]$. Each device is a microelectromechanical system (MEMS) which works by monitoring the modulation of the frequency of sound waves on a millimetre-sized piezoelectric substrate. The small size of SAW sensors allows them to react rapidly to changes in environmental parameters. Furthermore, the use of frequency modulation allows them to communicate accurate measurements which are largely unaffected by signal attenuation [12]. The benefits of using SAW sensors for displacement monitoring over other measurement techniques such as fibre-optic sensing [13] include their wireless capability, lower cost for mass production [14], higher durability under bending and reduced complexity for installation [15].

SAW sensors have historically been used to monitor aircraft component cracking [16], rebar corrosion [17], and tyre pressure [18], but their application to strain and displacement monitoring in civil structures, particularly in concrete, has been limited. The reasons for this include the fact that the brittleness of SAW sensors significantly limits their measurement range [19]. There are also challenges associated with bonding the sensors to the non-uniform and low precision geometries of concrete structures [20]. Even if these issues are overcome, concrete's heterogeneous nature means that the highly localised strain measurements provided by SAW sensors may not even provide valid indicators of global structural behaviour [21].

In this work, we demonstrate that SAW devices can be packaged to provide valid measurements of surface displacements and cracking in new or existing concrete structures. Tuning of the sensor's strain sensitivity and gauge-length allows us to obtain displacement measurements of a reinforced concrete beam under bending up until failure. We furthermore verify the accuracy of all measurements using computational methods and high-resolution optical-fibre-based strain monitoring techniques. By setting out a rigorous method for sensor testing in this work, it is hoped that the development of SAW sensors for concrete health monitoring may be fast tracked in future.

The paper begins with a brief outline of the measurement requirements and operating principles of the sensors in Section 2. The methods for designing, characterising and verifying sensor performance are then outlined in Section 3. The results of applying the sensor to monitor strain and cracking in a reinforced concrete beam are presented in Section 4, before the limitations and future work are discussed in Section 5.

\section{Theory and sensing principles}

In this work, we aim to design wireless SAW devices which can monitor crack opening in reinforced concrete structures until failure. To demonstrate the concept, SAW sensors will be used to monitor the surfacedisplacements of a singly-reinforced concrete beam under three-point bending.

\subsection{Reinforced concrete beam under bending}

As bending loads are applied to a reinforced concrete beam, brittle fracture occurs in the lower, tensile face of the beam and a distribution of cracks emerge [22]. The boundary layer below the steel reinforcement loses its strength and stiffness, resulting in the onset of a nonlinear deflection response. As the loads are increased further, the reinforcement holds the concrete together until the steel begins to yield at a nominal load given by [23]:

$F_{f}=\frac{A_{s} \sigma_{y}}{L}\left(d_{r}-\frac{a}{2}\right)$.

Here $L=60 \mathrm{~cm}$ is the loaded length of beam, while $A_{s}$ and $\sigma_{y}$ are the effective area and yield strength of the reinforcement respectively. The depth of the reinforcement, $d_{r}$, is measured from the compressed face of the beam during bending. In this work, a beam of width $w=10 \mathrm{~cm}$, and height $h=10 \mathrm{~cm}$ was cast from 
$\sigma_{c}=30 \mathrm{MPa}$ strength concrete. Two $8 \mathrm{~mm}$ diameter steel rebars were placed at depth of $d_{r}=8 \mathrm{~cm}$ during casting. Substitution of steel's yield strength, $\sigma_{y}=$ $500 \mathrm{MPa}$, suggests that the beam will completely fail at $F_{f}=12 \mathrm{kN}$.

The maximum displacement between two measurement points, $x_{A}$ and $x_{B}$, on the tensile face of the concrete beam will occur when the beam fails. Assuming the strain transfer from the reinforcement to the concrete is perfect, this maximum displacement will be:

$D_{m}=\varepsilon_{m}\left(x_{B}-x_{A}\right)=\frac{\sigma_{y}}{E_{s}}\left(\frac{h-C_{N A}}{d-C_{N A}}\right)\left(x_{B}-x_{A}\right)$,

where $E_{s}=200 \mathrm{GPa}$ is the elasticity of the steel reinforcement and $\varepsilon_{m}$ is the maximum strain induced in a displacement sensor which is mounted at points $x_{A}$ and $x_{B}$. The depth of the neutral axis, at which the strain in the beam is zero, is given by [24]:

$C_{N A}=\frac{A_{s} \sigma_{y}}{0.85^{2} \sigma_{c} w}$.

Substitution of equation (3) into (2) suggests that the maximum strain in a surface-mounted sensor at beam failure will be $\varepsilon_{m} \approx 3.5 m \varepsilon$. For a displacement sensor, of length $L_{s}=\left(x_{B}-x_{A}\right)=30 \mathrm{~cm}$, the maximum displacement is $D_{m} \approx 1 \mathrm{~mm}$. The aim in this work is therefore to design SAW sensors which can be retrofitted to concrete and used to monitor this strain/displacement range.

\subsection{Surface acoustic wave sensors}

The surface acoustic wave (SAW) sensors used in this work are one-port resonators. As shown in Figure 1, these are mainly comprised of a $9 \mathrm{~mm} \times 5 \mathrm{~mm}$ crystalline specimen of $0.35 \mathrm{~mm}$ thick, ST-cut quartz. A set of two interlinking metal forks, known collectively as an interdigitated tranducer (IDT), are deposited onto the plane-polished surface of the substrate [25]. As the quartz is piezoelectric, rapidly alternating the charge across the forks of the IDT generates local strain and compression. This produces a surface acoustic wave which resonates between two reflective gratings (metal strips or grooves), either side of the IDT. The resonant frequency of the acoustic wave is [26]:

$f_{0}=\frac{v_{p}}{p}$

where $p \approx 10 \mu \mathrm{m}$ is the pitch of the IDT (or gratings) and $v_{p}=3158 \mathrm{~m} / \mathrm{s}$ is the speed (or phase velocity) of the sound wave.

The device's pitch is increased by strains, $\varepsilon_{y}$, which separate the gratings. As quartz has an almost negligible Poisson ratio [27] and a low thermal expansion $\left(\alpha_{q}=3 \times 10^{-7}\right)$, the pitch is generally insensitive to orthogonal strains and changes in

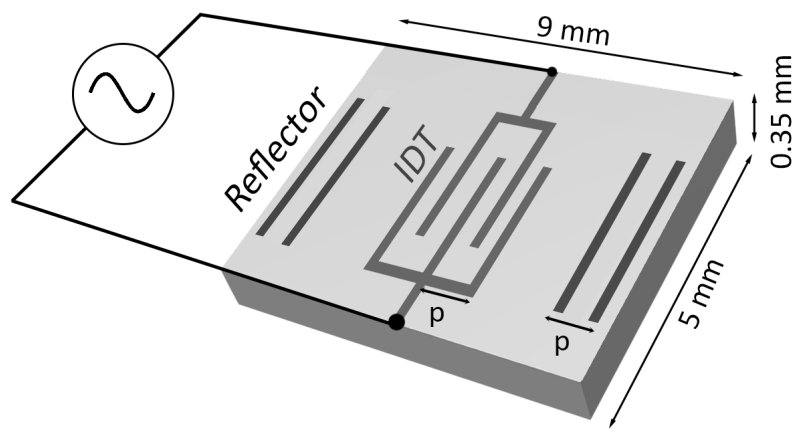

Figure 1. One-port resonator SAW sensor layout. The pitch, $p$, of the input-output IDT and the reflectors is labelled along with the dimensions of the quartz substrate.

temperature. The phase velocity, however, is sensitive to temperature shifts, $\Delta T$, due to the thermaldependence of quartz's material properties, such as its elasticity [28]. The overall sensitivity of a SAW device's resonant frequency to changes in strain and temperature therefore takes the form [29]:

$\frac{\Delta f_{0}}{f_{0}}=C_{\varepsilon} \varepsilon_{y}+g(\Delta T)$.

Here $C_{\varepsilon}=-0.95$ is the linear strain sensitivity of the SAW sensor. The temperature sensitivity is not linear, but can be described by a second-order polynomial:

$g(\Delta T)=C_{T 1} \Delta T+C_{T 2} \Delta T^{2}$,

where $C_{T 1} \sim 10^{-2} /{ }^{\circ} \mathrm{C}$ and $C_{T 2} \sim 10^{-2} /{ }^{\circ} \mathrm{C}^{2}$ are first and second order temperature coefficients respectively [29] . As the values of these coefficients are highly dependent on the manufacturing methods and materials used to fabricate the sensors, they are explicitly characterised in Section 3.

After the surface acoustic wave begins to resonate, the IDT also functions as a measurement node, converting the acoustic energy back into a measurable, electronic signal. One of the main benefits of SAW sensors is that they are passive devices. Rather than using a wired connection to the IDT, SAW sensors can be excited and read using wireless power delivery methods, such as RFID antennas.

\subsection{Fibre Bragg gratings}

Optical fibre Bragg grating (FBG) sensors have a proven track record of providing accurate, highresolution measurements of strain in structural health monitoring applications $[30,31]$. While FBGs are not a wireless technology, they were chosen to verify SAW sensor strain measurements in this work. An FBG is a $10 \mathrm{~mm}$ long periodic modulation in the refractive index of an optical fibre, which reflects a narrow distribution of wavelengths when it is illuminated using 
a broadband light source [32]. The centre of this wavelength distribution, termed the Bragg peak, $\lambda_{B}$, undergoes linear fractional shifts as strain, $\varepsilon$, and temperature changes, $\Delta T$, are applied to the FBG [32]:

$\frac{\Delta \lambda}{\lambda}=K_{\varepsilon} \varepsilon+K_{T} \Delta T$.

Here $K_{\varepsilon} \approx 0.8 \mathrm{ppm} / \mu \varepsilon$ and $K_{T} \approx 6.5 \mathrm{ppm} /{ }^{\circ} \mathrm{C}$ are the strain and temperature sensitivity of the FBG, respectively. The diameter of FBG sensors is around $125 \mu \mathrm{m}$. This small physical size is key in this work, as it prevents the sensors from perturbing the strain fields of the components they are monitoring and allows the FBGs to be attached alongside the SAW sensors.

\subsection{Temperature compensation}

It is clear from equations (5) and (7), that SAW and FBG sensors are sensitive to both strain and temperature changes. To monitor strains independently, these effects can be decoupled by monitoring local temperatures using a second, adjacent sensor which is isolated from strain. As ST-cut quartz SAW devices are inherently temperature stable at room temperature [33], it could be argued that temperature compensation is less crucial for shortterm measurements in ambient conditions. However, if SAW sensors are bonded to another component, then frequency shifts may be enhanced by the additional thermal expansion of this component, as demonstrated in Section 3.2.

\subsection{Measurement resolution and range}

Measurement resolutions are limited by how well the interrogation system can resolve shifts in the resonant frequency of the SAW device or the wavelength of the FBG's Bragg peak. In this work, prior to any bonding, SAW sensor strain and temperature resolutions were $3 \mu \varepsilon$ and $6{ }^{\circ} \mathrm{C}$ respectively. FBG strain and temperature resolutions were $1 \mu \varepsilon$ and $0.1^{\circ} \mathrm{C}$ respectively.

Both SAW and FBG sensors are able to monitor between $-20{ }^{\circ} \mathrm{C}$ and $+80^{\circ} \mathrm{C}$, which is an adequate temperature range for most civil engineering applications. While FBG sensors can monitor strains as high as $3 m \varepsilon[34]$, SAW sensor strain ranges are limited by the strength of the quartz substrate to $0.5-1 m \varepsilon$ with more polished substrates demonstrating higher strengths [19]. For high strain monitoring applications, strength limitations can be overcome through sensor packaging design, as described in Section 3.3.

\section{Sensor design and characterisation}

In this work, the SAW sensor's packaging was designed to compensate for the mechanical limitations of the device to allow it to wirelessly monitor large surface displacements in a concrete structure. In this section, the framework for packaging design is outlined, along with computational and experimental methods of verifying the characterisation.

\subsection{Fabrication and interrogation}

The packaged and installed sensor is shown in Figure 2. As shown, the SAW sensor is epoxied to the centre of an $L_{s}=30 \mathrm{~cm}$ long, $b=11.5 \mathrm{~mm}$ wide and $d=0.65 \mathrm{~mm}$ thick carbon-steel shim. Two holes at either end of the shim allow $4 \mathrm{~mm}$ diameter bolts ( $25 \mathrm{~mm}$ long) to be inserted and joined to the shim via induction brazing. The bolts are then grouted into $6 \mathrm{~mm}$ diameter holes, drilled into the surface of a notched concrete beam (the notch was used to encourage cracking at the centre of the beam). Mounting the sensor at a depth of $25 \mathrm{~mm}$ ensures that strain can be transferred from the beam to the sensor even when the surface of the concrete has become spalled or cracked. An FBG sensor was epoxied adjacent to the SAW sensor to verify its strain measurements.

SAW sensors were wirelessly interrogated using an RFID antenna from distances of 1-2 metres at a rate of $1 \mathrm{~Hz}$. The SAW strain sensor had a base resonant frequency (at zero strain and $20^{\circ} \mathrm{C}$ ) of $f_{0}=434.5 \mathrm{MHz}$. The FBG sensor, which had a base wavelength of $\lambda_{B}$ $=1550 \mathrm{~nm}$, was monitored via a fibre connection and using a commercial optical interrogator at a rate of $2 \mathrm{~Hz}$.

\subsection{Temperature characterisation}

Prior to installation onto the concrete beam, the packaged sensor was placed into an environmental chamber, along with an unbonded FBG sensor and an unbonded SAW sensor. The chamber was heated from $0^{\circ} \mathrm{C}$ to $40^{\circ} \mathrm{C}$ in $5{ }^{\circ} \mathrm{C}$ steps. The temperature was held for 2 hours at each step to allow the chamber to reach stable thermal equilibrium.

Figure 3 shows the response of each sensor as a function of the temperature shift from $0^{\circ} \mathrm{C}$ (measured using a local thermocouple). After epoxying to the shim, the SAW sensor's temperature response is only weakly non-linear, due to the large influence of the linear thermal expansion of the shim. Indeed, the enhancement to the FBG's temperature sensitivity after epoxying $\left(\Delta k_{T} \approx 10 \mathrm{ppm} /{ }^{\circ} \mathrm{C}\right)$ confirms this. The influence of bonding to steel is not as pronounced in the SAW sensor because the strain transfer from the shim to the quartz substrate is low, as discussed in Section 


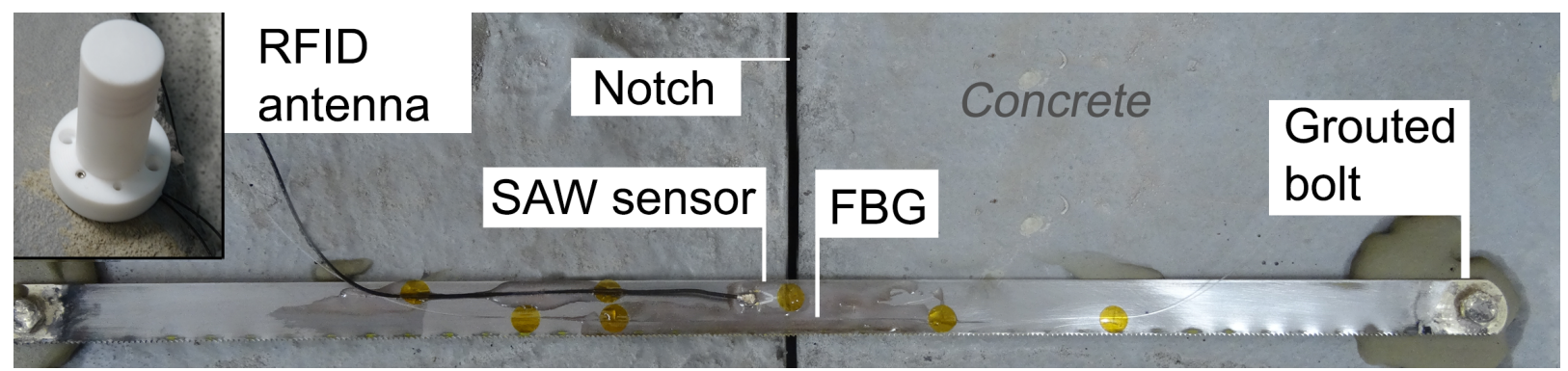

Figure 2. The bottom (tensile surface during bending) face of the notched concrete test beam, showing the sensor packaging (which is comprised of a carbon-steel shim and two steel bolts), grouted into the beam. The wireless SAW sensor, RFID antenna and the FBG used for measurement verification are labelled.
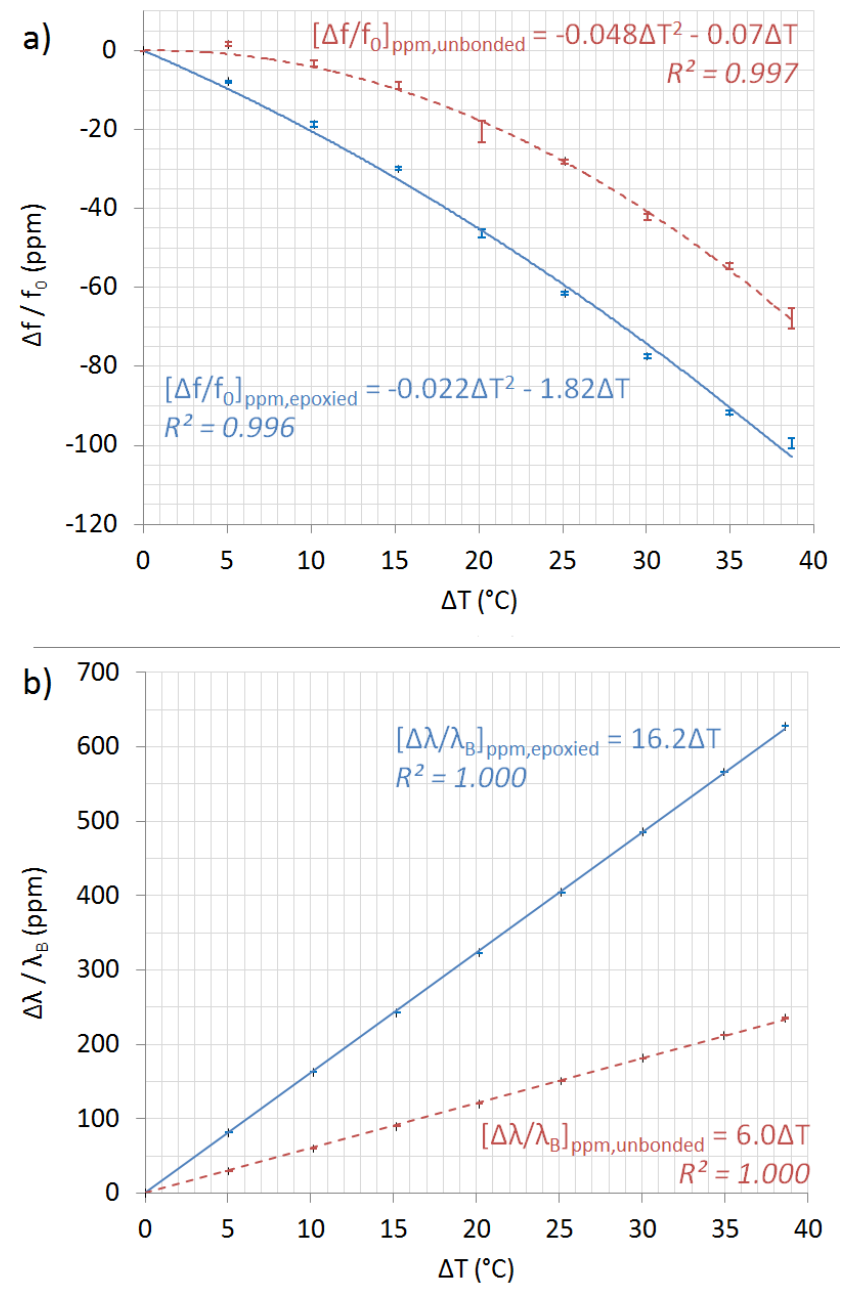

Figure 3. Temperature dependence of a) SAW sensor frequency and b) FBG sensor wavelength, both before and after epoxying to the steel shim. Fractional temperature shifts are measured relative to a datum of $\mathrm{T}=0{ }^{\circ} \mathrm{C}$.

3.3.1. Nevertheless, the added expansion of the steel improves the SAW sensor's temperature resolution to $2{ }^{\circ} \mathrm{C}$.

\subsection{Strain characterisation}

To characterise strain sensitivity prior to installation onto the concrete beam, the bolts of the sensor packaging were clamped and masses of up to $M=$ $0.3 \mathrm{~kg}$ were hung from the centre of the shim to induce three-point bending. In this set up, the maximum strain in the shim is:

$\varepsilon_{\max }=\left(\frac{M g L_{s}}{4}\right)\left(\frac{d}{I_{M} E_{s t}}\right)$

where $g=9.81 \mathrm{~m} / \mathrm{s}^{2}$ is graviational acceleration, $I_{M}=$ $b d^{3} / 12$ is the beam's second moment of area and $E_{s t} \approx$ $200 \mathrm{GPa}$ is its elasticity.

In practice, empirical measurements of $I_{M}$ and $E_{s t}$ are challenging, as they require accurate knowledge of the shim's geometry and stiffness. However, $I_{M} E_{s t}$ can also be derived from the shim's resonant frequency, $\omega_{c}$, when it is allowed to oscillate as a fixed-free cantilever: $I_{M} E_{s t}=\frac{\omega_{c}^{2} L_{s}^{3}}{3}\left(0.236 M_{\text {shim }}+M_{\text {bolt }}\right) \approx(51 \pm 1) \times 10^{-3}$.(9) Here $M_{\text {shim }}=17.9 \mathrm{~g}$ and $M_{\text {bolt }}=2.7 \mathrm{~g}$ are the masses of the shim and bolt respectively. To find $\omega_{c}=$ $28.53 \mathrm{rad} / \mathrm{s}$, a high-speed interrogator was briefly used to monitor the frequency of strain oscillations in the epoxied FBG at a rate of $3 \mathrm{kHz}$.

For the three-point bending test, Figure 4 shows the strains measured by the two sensors (derived using equations (5) and (7)), plotted as a function of the applied shim strain, $\varepsilon_{\max }$. Both sensors respond linearly, but the SAW sensor's strain transfer was only $33 \%$ of that of the FBG sensor. Furthermore, the strain transfer to the FBG itself was surprisingly low, as epoxied FBG sensors typically show strain transfers of 0.9 or above [35].

3.3.1. FEM investigation To investigate the strain transfer issue further, a finite element model (FEM) of the sensor packaging under bending was constructed. As shown in Figure 5, during load case 1, the shim was modelled independently, while in load case 2, a quartz SAW sensor was perfectly-bonded to the shim. 
a) Load case 1: shim only

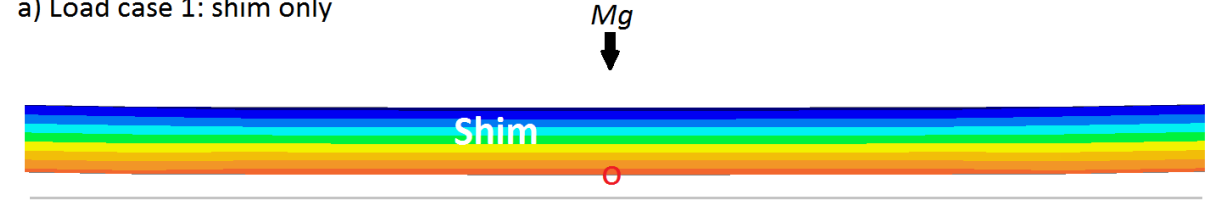

b) Load case 2 : shim and SAW
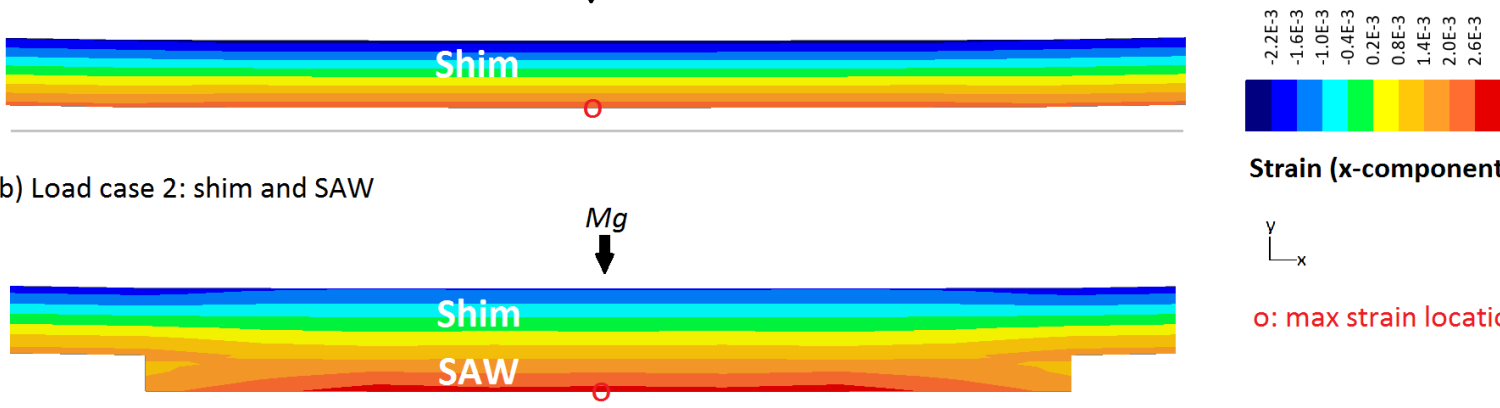

Strain (x-component)

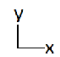

o: max strain location

Figure 5. FEM of the axial strain, $\varepsilon_{x}$, for a) load case 1: the shim only and b) load case 2: the shim bonded to the SAW sensor, under a three-point bending force of $M g=5 \mathrm{~N}$. Only the region near the centre of the shim is shown, and the location of maximum strain in the model circled for each case.

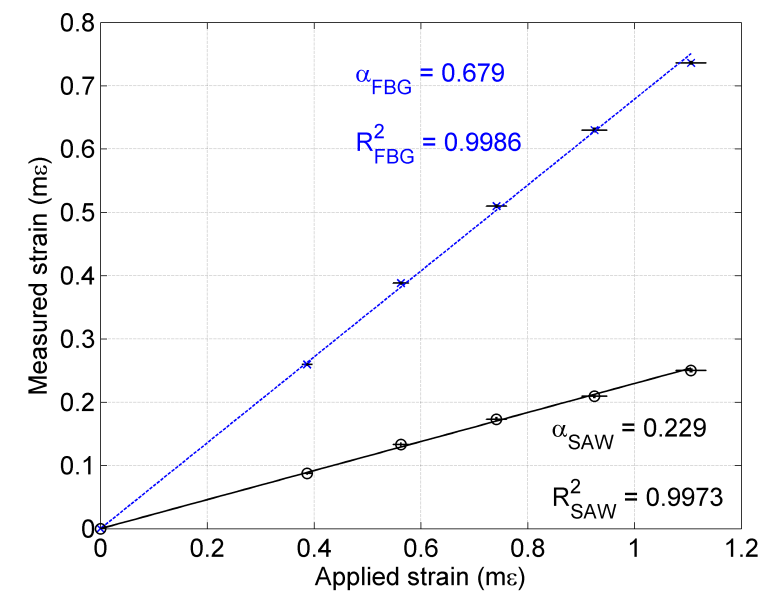

Figure 4. The strain measured by the FBG and SAW sensors as a function of the bending strain applied to the steel shim housing. The strain transfer coefficients and goodness of fit $\left(R^{2}\right.$ values $)$ are also shown. Due to fluctuations in ambient temperature, average measurement errors for the SAW sensor were $1.5 \%$, while those for the FBG sensor were $0.5 \%$.

Figure 6 shows the axial strain, normalised by $\varepsilon_{\max }$, as a function of model thickness, $y$. In loadcase 1 , the FEM's maximum strain is $2.4 m \varepsilon$, which is within $\pm 5 \%$ agreement with equation (8). This demonstrates the validity of the FEM. When the SAW sensor is added to the model in load case 2, the maximum strain at the surface of the shim decreases by around $50 \%$ (and the results suggest that this decrease acts over the entire width of the shim). The result confirms that the FBG's strain has been diminished in Figure 4 because the SAW sensor's finite thickness has contributed to a higher stiffness, causing it to interfere with the shim's strain field. The drop in strain is, however, more severe in the FEM because perfect bonding between the SAW and the shim is assumed.

Surface acoustic waves are affected by the bulk

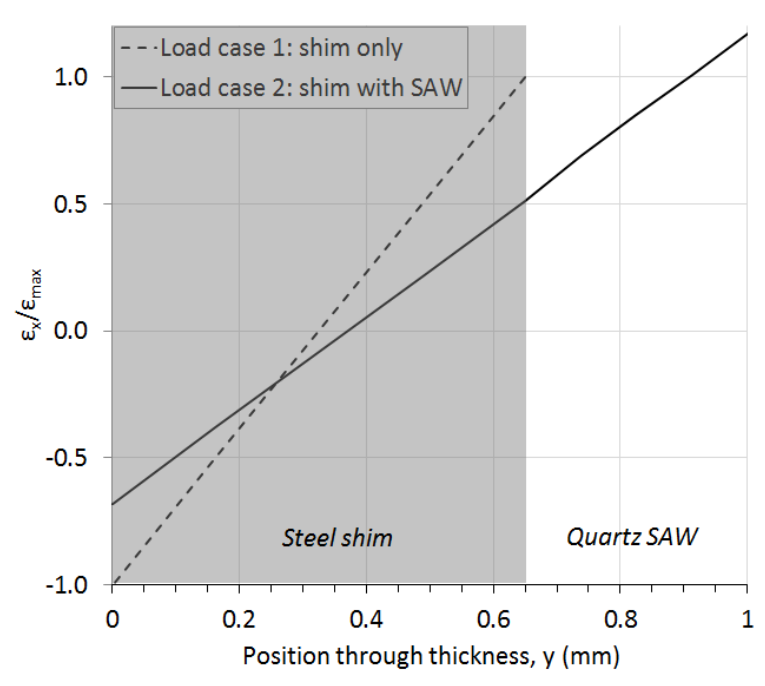

Figure 6. Normalised strain plotted as a function of thickness, $y$ through the FEM. The strains are plotted for loadcase a) shim only, and b) shim with SAW, which are defined in Figure 5. The boundaries of the steel shim are shown by the shaded region on the graph.

properties of the quartz substrate, and Figure 5 highlights that the SAW sensor is subjected to a distribution of strains across its thickness. The strain field over the SAW sensor's thickness varies from $0.5 \varepsilon_{\max }$, to $1.1 \varepsilon_{\max }$. This partly explains the lower measured strains of the SAW sensor in Figure 4. In this work, the SAW sensor was bonded from one side to the shim, but fully encapsulating the sensors in epoxy may enhance the strain transfer. Misalignments or cracking in the epoxy layer during initial characterisation may also have further reduced strain transfer.

3.3.2. Strain transfer after installation After attachement of the packaged sensor to the concrete beam, the strain transferred to the sensors is further reduced. As 


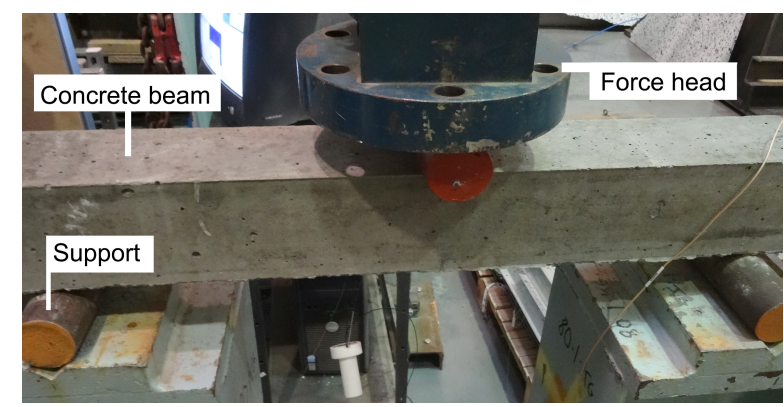

Figure 7. The reinforced concrete beam under three-point bending, showing the supports and the force head.

the shim is $L_{s}=30 \mathrm{~cm}$ long, its strain after grouting to the concrete beam is described by:

$\varepsilon_{s}=\frac{\Delta L_{s}}{L_{s}}=\frac{u_{B}-u_{A}}{x_{B}-x_{A}}=\frac{1}{L_{s}} \int_{x_{A}}^{x_{B}} \varepsilon(x) d x+\frac{1}{L_{s}} \sum_{j} \Delta w_{j}$.

Here $u_{A, B}$ are the displacements of the anchoring bolts, which are grouted into the concrete beam at locations $x_{A, B}$. The quantities $\Delta w_{j}$ are a set of added strain discontinuities caused by the concrete cracking [21]. The integral in equation (10) shows that the sensor housing provides an average measurement of the concrete strain between its two bonding points, resulting in a reduced strain and spatial resolution. Long-gauge sensing is, however, required in this case because concrete is an inhomogenous composite material. Monitoring over small length scales may provide measurements of single crack growth, but longgauge sensors allow multiple cracks and bulk properties to be measured.

As the gauge length is approximately half of the length of the concrete beam, the strain transfer should be reduced by approximately half during three-point bending (ignoring any non-linear effects). The overall strain transfer from the concrete beam to the SAW sensor should therefore be $\alpha_{S A W} / 2 \approx 0.1$. This means that the $3.5 \mathrm{m \varepsilon}$ sensor strain limit derived in Section 2.1 should not breach the $500 \mu \varepsilon$ strain limit of the SAW sensor during beam testing. It does, however, also mean that the packaged SAW sensor's strain resolution is reduced from $3 \mu \varepsilon$ to $30 \mu \varepsilon$. This is equivalent to reducing the displacement resolution from $1 \mu \mathrm{m}$ to $10 \mu \mathrm{m}$.

\section{Application and results}

The instrumented concrete beam was placed into a three-point bend testing rig, with supports which were $60 \mathrm{~cm}$ apart, as shown in Figure 7 . Integrated transducers monitored the stroke (displacement) and applied load of a force head used to deflect the beam. In the first set of experiments, time-varying strokes of frequency $0.05 \mathrm{~Hz}$ and amplitude $0.1,0.25,0.5,0.75$

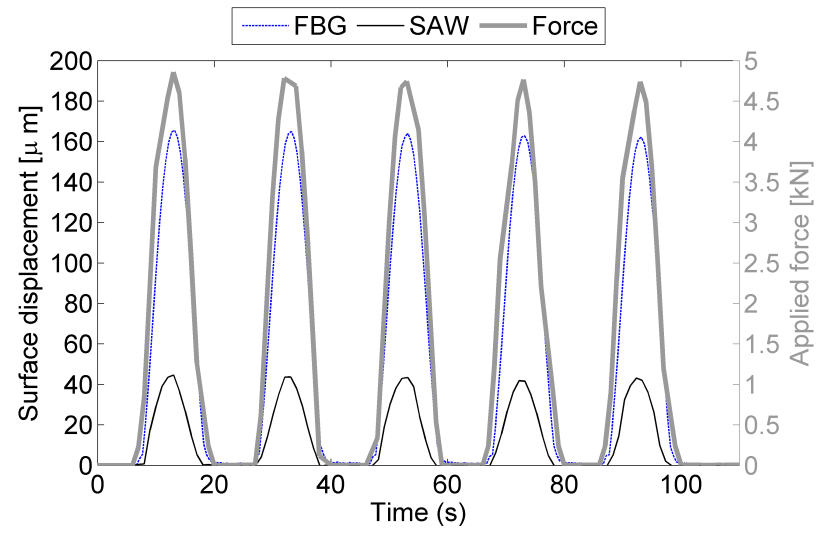

Figure 8. The surface displacements measured by the FBG and SAW sensors during a $4 \mathrm{~mm}$ stroke (approximately $5 \mathrm{kN}$ force) cyclic bending test. and $1 \mathrm{~mm}$ were applied to the concrete beam. In the second experiment, the load was ramped linearly at a rate of $0.05 \mathrm{kN} / \mathrm{s}$ up until the first signs of beam failure. The load was then held for 90 seconds, before the test was continued at a constant stroke rate of $0.05 \mathrm{~mm} / \mathrm{s}$. This ensured that the beam underwent gradual failure, to capture as much information as possible.

Throughout all tests, a local, unbonded FBG was used as a strain-isolated reference for both SAW sensor and FBG strain sensor measurements, allowing temperature effects to be decoupled.

\subsection{Cyclic loading}

An example of the sensor responses during a $4 \mathrm{~mm}$ cyclic stroke displacement are shown in Figure 8. Although the SAW sensor has a reduced response, it is still able to accurately resolve the time-varying surface displacements in the tensioned surface of the beam.

The peak surface displacement measured during all cyclic tests is plotted against the peak applied force in Figure 9. Non-linear displacement behaviour due to cracking of the beam can clearly be seen. The strain transfer to the SAW sensor has decreased slightly as it now measures strains which are $28 \%$ of the strain in the FBG (rather than the $33 \%$ found in Section 3.3). This reduction is possibly due to microcracking, ageing and relaxation of the epoxy layer used to attach the SAW sensor, as its reasonably large size can cause high stresses at the packaging interface.

\subsection{Linear force ramp to failure}

A linear force ramp was applied to the concrete beam at a rate of $0.05 \mathrm{kN} / \mathrm{s}$ up until the first signs of beam failure at $9 \mathrm{kN}$. The load was then held at $9 \mathrm{kN}$ for 90 seconds, before the force head was controlled at a stroke rate of $0.05 \mathrm{~mm} / \mathrm{s}$ up until complete beam 


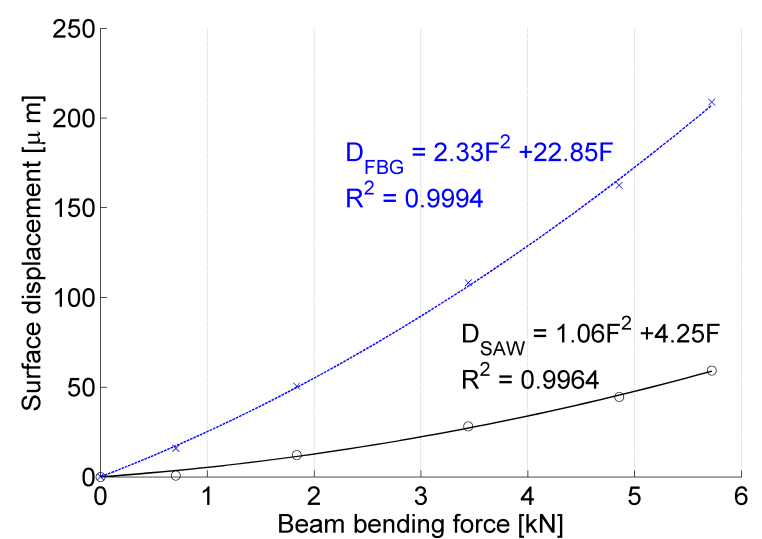

Figure 9. The surface displacements by the FBG and SAW sensors as a function of the cyclic force applied to the concrete beam. The second-order dependence of the displacement on the force arises due to concrete's non-linear behaviour.

failure. The applied force as a function of the stroke is shown in Figure 10. After the apparatus has resolved initial contact forces, the beam transitions through a linear region. This linear regions lasts up until $5 \mathrm{kN}$, as the prior cyclic loading has already induced cracking up to this level. The response is therefore apparently elastic as the force is taken up by the rebars. Beyond $5 \mathrm{kN}$, the beam begins to behave non-linearly, before failing at a force of $12.6 \mathrm{kN}$. This value broadly agrees with the theoretical failure force of $12 \mathrm{kN}$ derived in Section 2.1.

The SAW sensor response, FBG sensor response and applied force during this test are shown as a time series in Figure 11. The scattered points, obtained by interpolating from Figure 9, are also shown for reference. The agreement between the cyclic and linear ramp tests demonstrates sensor repeatability. However, it is clear that the response during the force ramp test is more linear as the pre-cracked concrete is contributing less to the beam's stiffness, so the surface displacements under bending forces are larger.

Beyond an applied force of $7 \mathrm{kN}$, both sensors measure a reduced surface displacement, even though the stroke rate in Figure 10 is increasing. This is because, at higher loads, the anchorages at the location of the grouted bolts become a defect for crack propagation. This reduces the strain applied to the sensor packaging. At $9 \mathrm{kN}$ (180 seconds into the test) the beam showed significant cracking, and so the force was held. Both the SAW and FBG sensors remained stable during the time period. The beam was finally taken to failure in stroke mode. The sensors show some increase in measured surface displacement before the cracking through the anchorages begins to reverse the strains in the packaging. Note that, throughout the test, the SAW sensor did not exceed its $500 \mu \varepsilon$ limit

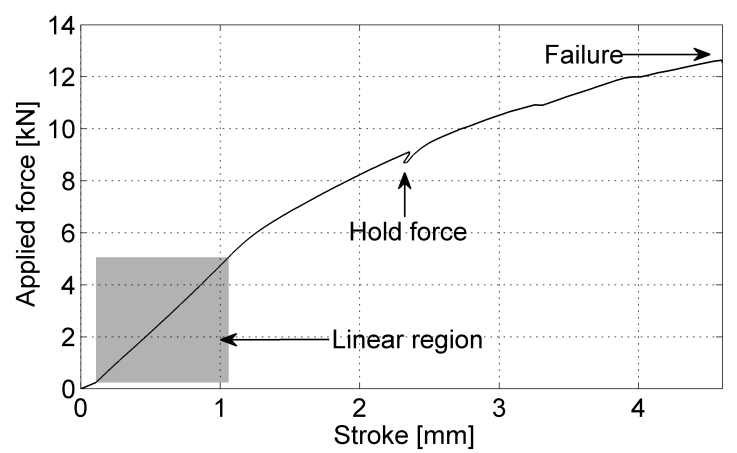

Figure 10. Applied force as a function of stroke (force head displacement) during the linear ramp to failure. The region of linear behaviour is highlighted, along with the force at which the beam was held for 90 seconds, and the point of beam failure.

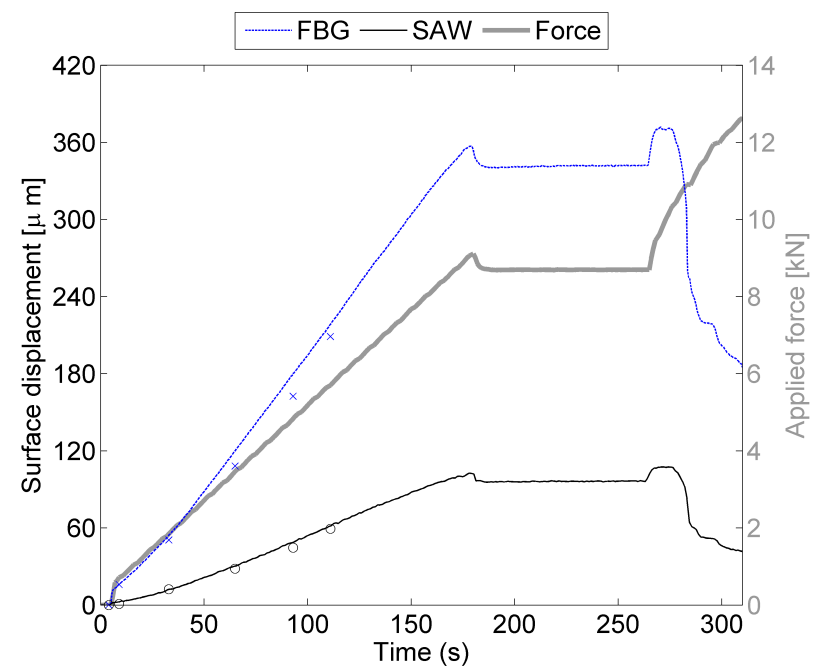

Figure 11. The force and measured surface displacements during the linear force ramp. At 180 seconds, the force was held constant for 90 seconds to demonstrate sensor stability. Beyond this the beam was deflected in constant stroke mode. The scatter points shown are derived from Figure 10, and demonstrate that the sensor behaviour is quite repeatable.

and so remained operational.

\section{Discussion}

In this work, we demonstrate that sensor packaging can be used to overcome the mechanical limitations of SAW sensors to allow them to provide wireless strain measurements of reinforced concrete, even up until its failure. By tuning the length of the packaging, we were able to retrofit sensors to allow the bulk strains of a reinforced concrete beam to be monitored. The sensor could be conveniently characterised and verified prior to installation, so its behaviour after installation was understood. 
While details are not reported here, several other methods of surface-mounting SAW sensors onto existing reinforced concrete members were explored in this work. During these feasibility studies, the metrics used to gauge the suitability of each method were:

- Installation - procedures for installation should be as rapid and convenient as possible and suitable for varying weather conditions.

- Invasiveness - sensor anchorages should not penetrate any deeper than typical concrete covers over rebars. Any drilled anchorages should be of a small diameter.

- Reliability — sensors should be robust and maintain an adequate strain transfer and range.

Epoxy-bonding the SAW sensors to the concrete surface was an obvious and minimally invasive solution. However, as this does not extend the gauge length of the sensors, it only allows for reliable monitoring over a $10 \mathrm{~mm}$ length of a pristine concrete surface. Achieving a reliable bond also required a fully cured primer layer of epoxy, prior to a secondary layer of epoxy to bond the sensor. The method was therefore not only time consuming, but also unsuitable for cold and wet weather conditions. The grouted anchorage method, described in this paper, is more invasive than epoxying, but is suitable for structures with at least $30 \mathrm{~mm}$ of concrete cover (which is the minimum recommended by most building standards [23]). The use of an accelerated grout also makes the method suitable for rapid installation in wet and cold conditions. At room temperatures, the grout was set within minutes and could be fully loaded within a few days. While the bolts did provide the point of failure in the beam tests, this was due to the large size of the holes relative to the beam thickness and applied loads, and may not pose an issue for larger structure.

SAW sensors naturally lend themselves to multiplexing. The initial frequency of SAW devices can be customised during fabrication so that frequencydivision-multiplexed arrays of SAW devices can be used to monitor quasi-distributed strains, displacements and temperatures in large structures with a single interrogation device. Even more sensors can be interrogated with a single interrogator if time and codedivision multiplexing are used [36]. The advantage of wireless sensing over FBG technology is that the physical complexity of the interrogation network is reduced: considerations about where to trail fibres or wires are removed. However, as with any wireless device, achieving an efficient coupling between the transmitting and receiving antennas is essential. As SAW technology utilises RFID, the wireless interrogation distance is typically limited to a few metres in air, but signals are dramatically attenuated by concrete, soil or metal. As such, SAW devices are not suitable for monitoring concrete assets below ground, or those in steel-congested areas.

While the strain/displacement measurement range was increased in this work, the trade-off was a reduction in measurement sensitivity. If the application demands a higher resolution, then this can be facilitated by reducing the thickness of the SAW device or by reducing the gauge length of the sensor. In this work, thermal and strain effects were assumed to act independently, and this is not an atypical approach for sensor characterisation. However, as SAW sensors operate based on changes on both phase velocity and pitch, strain sensitivity will likely have some dependence on operating temperature, and viceversa. In an environment with large fluctuations in both strain and temperature, these effects should be accounted for [37].

\section{Conclusions}

We have demonstrated that wireless surface acoustic wave sensors can be used for monitoring large (2$4 m \varepsilon$ ) strains and crack opening of up to $1 \mathrm{~mm}$ during cyclic and static loading of reinforced concrete structures. The strain accuracy of the SAW sensor measurements was verified using computational models and a secondary fibre-based strain sensor. By careful design of the packaging, the strain transfer from the concrete beam to the SAW sensor could be reduced to around 10-20\% to prevent the SAW device's quartz substrate from reaching its fracture limit during large structural deflections. The technology may now be suitable for retrofitting to existing concrete structures to wirelessly monitor surface displacements and crack opening.

\section{References}

[1] Spencer B F, Ruiz-Sandoval M E and Kurata N Struct. Control Health Monit. 11 349-368

[2] Lynch J P and Loh K J Shock Vib. Dig. 38 91-128

[3] Cho S, Spencer B F, Jo H, Li J and Kim R E Bridge monitoring using wireless smart sensors SPIE Newsroom

[4] Kotowsky M, Dowding C and Fuller K Wireless sensor networks to monitor crack growth on bridges Developing a Research Agenda for Transportation Infrastructure Preservation and Renewal

[5] Lynch J P Philos. Trans. R. Soc. A 365 345-372

[6] Popovici E, Magno M and Marinkovic S Power management techniques for wireless sensor networks: A review 5th International Workshop on Advances in Sensors and Interfaces pp 194-198

[7] White R New prospects for acoustic sensors: an overview 41st Annual Symposium on Frequency Control. 1987 pp 333-338

[8] Varadan V K and Varadan V V Smart Mater. Struct. 9953

[9] Kalinin V Passive wireless strain and temperature sensors based on SAW devices IEEE Radio and Wireless Conference pp 187-190 
[10] Drafts B IEEE Trans. Microw. Theory Tech. 49 795-802

[11] Wolff U, Dickert F L, Fischerauer G K, Greibl W and Ruppel C C W IEEE Sens. J. 1 4-13

[12] Buff W SAW sensors for direct and remote measurement IEEE Ultrasonics Symposium vol 1 pp 435-443

[13] Glisic B and Inaudi D Crack monitoring in concrete elements using long-gage fiber optic sensors

[14] Kalansuriya P, Bhattacharyya R and Sarma S IEEE Sens. J. 13 1564-1570

[15] Sahay P, Kaya M and Wang C 13 39-57

[16] Kim J S, Vinoy K J and Varadan V K Wireless health monitoring of cracks in structures with MEMS-IDT sensors SPIE's 9th Annual International Symposium on Smart Structures and Materials vol 4700 pp 342-353

[17] Li Z, Li F, Zdunek E, Landis E and Shah S ACI Mater. J. 95

[18] Zhang X, Wang F, Wang Z, Li W and He D Intelligent tires based on wireless passive surface acoustic wave sensors 7 th International IEEE Conference on Intelligent Transportation Systems pp 960-964

[19] Chao H and Parker T Tensile fracture strength of ST cut quartz 37th Annual Symposium on Frequency Control. 1983 pp $116-124$

[20] Chang P C, Flatau A and Liu S C Structural Health Monitoring 2 257-267

[21] Glisic B Meas. Sci. Technol. 22035206

[22] Hwang L and Rizkalla S Effective tensile stress-strain characteristics for reinforced concrete Proceedings of the Canadian Society of Civil Engineering pp 129-147

[23] American Concrete Institute Building code requirements for structural concrete (ACI 318), ACI committee 318

[24] Whitney C S ACI J. Proc. 33

[25] Shiokawa S and Kondoh J Jpn. J. Appl. Phys. 432799

[26] Springer A, Weigel R, Pohl A and Seifert F 9 745-756

[27] Levien L, Prewitt C and Weidner J Am. Mineral. 65920 930

[28] Moller F and Kuhn J Sensors and Actuators A: Physical $3073-75$

[29] Ballantine D S, White R M, Martin S J, Ricco A J, Zellers E T, Frye G C and Wohltjen H Acoustic Wave Sensors: Theory, Design, \& Physico-Chemical Applications (Academic Press) ISBN 978-0-08-052333-0

[30] Majumder M, Gangopadhyay T K, Chakraborty A K, Dasgupta K and Bhattacharya D K Sensors and Actuators A: Physical 147 150-164

[31] Leung C K Y, Wan K T, Inaudi D, Bao X, Habel W, Zhou $\mathrm{Z}$, Ou J, Ghandehari M, Wu H C and Imai M Mater Struct 48 871-906

[32] Hill K O and Meltz G J. Light. Technol. 15 1263-1276

[33] Yamazaki T, Iizawa K, Kanna S and Takagi M Jpn. J. Appl. Phys. 423136

[34] Yoon H J and Kim C G Smart Material Structures 16 13151319

[35] Wan K T, Leung C K Y and Olson N G Smart Mater. Struct. 17035037

[36] Malocha D C, Gallagher M, Fisher B, Humphries J, Gallagher D and Kozlovski N 13 5897-5922

[37] Alzuaga S, Michoulier E, Friedt J M, Martin G, Berthelot $\mathrm{P}$, Ptrini $\mathrm{V}$ and Ballandras $\mathrm{S}$ Characterization of the thermal dependence of SAW stress sensitivity 10me Congrs Franais d'Acoustique 


\title{
Wireless surface acoustic wave sensors for displacement and crack monitoring in concrete structures
}

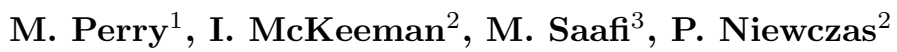 \\ 1 Department of Civil and Environmental Engineering, James Weir Building, \\ University of Strathclyde, Glasgow, G1 1XJ \\ 2 Department of Electronic and Electrical Engineering, Royal College Building, \\ University of Strathclyde, Glasgow, G1 1XW \\ 3 Department of Engineering, Lancaster University, Lancaster, LA1 4YR \\ E-mail: ${ }^{1} \mathrm{~m}$.perry@strath.ac.uk, ${ }^{3} \mathrm{~m}$.saafi@lancaster.ac.uk
}

\begin{abstract}
.
In this work, we demonstrate that wireless surface acoustic wave devices can be used to monitor millimetre displacements in crack opening during the cyclic and static loading of reinforced concrete structures. Sensors were packaged to extend their gauge length and to protect them against brittle fracture, before being surface-mounted onto the tensioned surface of a concrete beam. The accuracy of measurements was verified using computational methods and optical-fibre strain sensors. After packaging, the displacement and temperature resolutions of the surface acoustic wave sensors were $10 \mu \mathrm{m}$ and $2{ }^{\circ} \mathrm{C}$ respectively. With some further work, these devices could be retrofitted to existing concrete structures to facilitate wireless structural health monitoring.
\end{abstract}




\section{Introduction}

Rapid developments in wireless sensing have been driven by a desire for compact devices which allow for flexible and rapid installation [1]. Wireless sensor networks have already been successfully deployed in various structural health monitoring (SHM) applications $[2,3,4]$, but the limited battery life of wireless sensors typically leads to reduced performance levels, or increased costs from the development of data compression or energy harvesting methods $[5,6]$.

Surface acoustic wave (SAW) devices are a class of wireless sensor which require no batteries, as they operate on wireless power. By utilising radio-frequency identification (RFID) technology, SAW sensors can provide passive measurements of strain, temperature, pressure, acceleration and chemical contamination, even in harsh environments $[7,8,9,10,11]$. Each device is a microelectromechanical system (MEMS) which works by monitoring the modulation of the frequency of sound waves on a millimetre-sized piezoelectric substrate. The small size of SAW sensors allows them to react rapidly to changes in environmental parameters. Furthermore, the use of frequency modulation allows them to communicate accurate measurements which are largely unaffected by signal attenuation [12]. The benefits of using SAW sensors for displacement monitoring over other measurement techniques such as fibre-optic sensing [13] include their wireless capability, lower cost for mass production [14], higher durability under bending and reduced complexity for installation [15].

SAW sensors have historically been used to monitor aircraft component cracking [16], rebar corrosion [17], and tyre pressure [18], but their application to strain and displacement monitoring in civil structures, particularly in concrete, has been limited. The reasons for this include the fact that the brittleness of SAW sensors significantly limits their measurement range [19]. There are also challenges associated with bonding the sensors to the non-uniform and low precision geometries of concrete structures [20]. Even if these issues are overcome, concrete's heterogeneous nature means that the highly localised strain measurements provided by SAW sensors may not even provide valid indicators of global structural behaviour [21].

In this work, we demonstrate that SAW devices can be packaged to provide valid measurements of surface displacements and cracking in new or existing concrete structures. Tuning of the sensor's strain sensitivity and gauge-length allows us to obtain displacement measurements of a reinforced concrete beam under bending up until failure. We furthermore verify the accuracy of all measurements using computational methods and high-resolution optical-fibre-based strain monitoring techniques. By setting out a rigorous method for sensor testing in this work, it is hoped that the development of SAW sensors for concrete health monitoring may be fast tracked in future.

The paper begins with a brief outline of the measurement requirements and operating principles of the sensors in Section 2. The methods for designing, characterising and verifying sensor performance are then outlined in Section 3. The results of applying the sensor to monitor strain and cracking in a reinforced concrete beam are presented in Section 4, before the limitations and future work are discussed in Section 5.

\section{Theory and sensing principles}

In this work, we aim to design wireless SAW devices which can monitor crack opening in reinforced concrete structures until failure. To demonstrate the concept, SAW sensors will be used to monitor the surfacedisplacements of a singly-reinforced concrete beam under three-point bending.

\subsection{Reinforced concrete beam under bending}

As bending loads are applied to a reinforced concrete beam, brittle fracture occurs in the lower, tensile face of the beam and a distribution of cracks emerge [22]. The boundary layer below the steel reinforcement loses its strength and stiffness, resulting in the onset of a nonlinear deflection response. As the loads are increased further, the reinforcement holds the concrete together until the steel begins to yield at a nominal load given by [23]:

$F_{f}=\frac{A_{s} \sigma_{y}}{L}\left(d_{r}-\frac{a}{2}\right)$.

Here $L=60 \mathrm{~cm}$ is the loaded length of beam, while $A_{s}$ and $\sigma_{y}$ are the effective area and yield strength of the reinforcement respectively. The depth of the reinforcement, $d_{r}$, is measured from the compressed face of the beam during bending. In this work, a beam of width $w=10 \mathrm{~cm}$, and height $h=10 \mathrm{~cm}$ was cast from 
$\sigma_{c}=30 \mathrm{MPa}$ strength concrete. Two $8 \mathrm{~mm}$ diameter steel rebars were placed at depth of $d_{r}=8 \mathrm{~cm}$ during casting. Substitution of steel's yield strength, $\sigma_{y}=$ $500 \mathrm{MPa}$, suggests that the beam will completely fail at $F_{f}=12 \mathrm{kN}$.

The maximum displacement between two measurement points, $x_{A}$ and $x_{B}$, on the tensile face of the concrete beam will occur when the beam fails. Assuming the strain transfer from the reinforcement to the concrete is perfect, this maximum displacement will be:

$D_{m}=\varepsilon_{m}\left(x_{B}-x_{A}\right)=\frac{\sigma_{y}}{E_{s}}\left(\frac{h-C_{N A}}{d-C_{N A}}\right)\left(x_{B}-x_{A}\right)$,

where $E_{s}=200 \mathrm{GPa}$ is the elasticity of the steel reinforcement and $\varepsilon_{m}$ is the maximum strain induced in a displacement sensor which is mounted at points $x_{A}$ and $x_{B}$. The depth of the neutral axis, at which the strain in the beam is zero, is given by [24]:

$C_{N A}=\frac{A_{s} \sigma_{y}}{0.85^{2} \sigma_{c} w}$.

Substitution of equation (3) into (2) suggests that the maximum strain in a surface-mounted sensor at beam failure will be $\varepsilon_{m} \approx 3.5 \mathrm{m \varepsilon}$. For a displacement sensor, of length $L_{s}=\left(x_{B}-x_{A}\right)=30 \mathrm{~cm}$, the maximum displacement is $D_{m} \approx 1 \mathrm{~mm}$. The aim in this work is therefore to design SAW sensors which can be retrofitted to concrete and used to monitor this strain/displacement range.

\subsection{Surface acoustic wave sensors}

The surface acoustic wave (SAW) sensors used in this work are one-port resonators. As shown in Figure 1 , these are mainly comprised of a $9 \mathrm{~mm} \times 5 \mathrm{~mm}$ crystalline specimen of $0.35 \mathrm{~mm}$ thick, ST-cut quartz. A set of two interlinking metal forks, known collectively as an interdigitated tranducer (IDT), are deposited onto the plane-polished surface of the substrate [25]. As the quartz is piezoelectric, rapidly alternating the charge across the forks of the IDT generates local strain and compression. This produces a surface acoustic wave which resonates between two reflective gratings (metal strips or grooves), either side of the IDT. The resonant frequency of the acoustic wave is [26]:

$f_{0}=\frac{v_{p}}{p}$,

where $p \approx 10 \mu m$ is the pitch of the IDT (or gratings) and $v_{p}=3158 \mathrm{~m} / \mathrm{s}$ is the speed (or phase velocity) of the sound wave.

The device's pitch is increased by strains, $\varepsilon_{y}$, which separate the gratings. As quartz has an almost negligible Poisson ratio [27] and a low thermal expansion $\left(\alpha_{q}=3 \times 10^{-7}\right)$, the pitch is generally insensitive to orthogonal strains and changes in

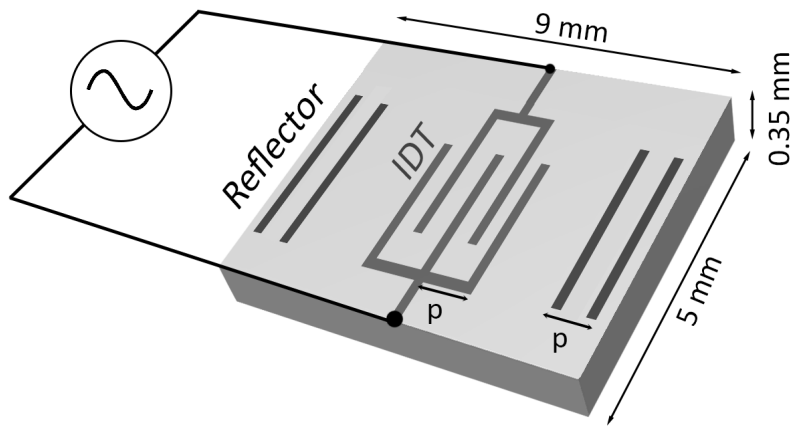

Figure 1. One-port resonator SAW sensor layout. The pitch, $p$, of the input-output IDT and the reflectors is labelled along with the dimensions of the quartz substrate.

temperature. The phase velocity, however, is sensitive to temperature shifts, $\Delta T$, due to the thermaldependence of quartz's material properties, such as its elasticity [28]. The overall sensitivity of a SAW device's resonant frequency to changes in strain and temperature therefore takes the form [29]:

$\frac{\Delta f_{0}}{f_{0}}=C_{\varepsilon} \varepsilon_{y}+g(\Delta T)$.

Here $C_{\varepsilon}=-0.95$ is the linear strain sensitivity of the SAW sensor. The temperature sensitivity is not linear, but can be described by a second-order polynomial:

$g(\Delta T)=C_{T 1} \Delta T+C_{T 2} \Delta T^{2}$,

where $C_{T 1} \sim 10^{-2} /{ }^{\circ} \mathrm{C}$ and $C_{T 2} \sim 10^{-2} /{ }^{\circ} \mathrm{C}^{2}$ are first and second order temperature coefficients respectively [29] . As the values of these coefficients are highly dependent on the manufacturing methods and materials used to fabricate the sensors, they are explicitly characterised in Section 3.

After the surface acoustic wave begins to resonate, the IDT also functions as a measurement node, converting the acoustic energy back into a measurable, electronic signal. One of the main benefits of SAW sensors is that they are passive devices. Rather than using a wired connection to the IDT, SAW sensors can be excited and read using wireless power delivery methods, such as RFID antennas.

\subsection{Fibre Bragg gratings}

Optical fibre Bragg grating (FBG) sensors have a proven track record of providing accurate, highresolution measurements of strain in structural health monitoring applications [30, 31]. While FBGs are not a wireless technology, they were chosen to verify SAW sensor strain measurements in this work. An FBG is a $10 \mathrm{~mm}$ long periodic modulation in the refractive index of an optical fibre, which reflects a narrow distribution of wavelengths when it is illuminated using 
a broadband light source [32]. The centre of this wavelength distribution, termed the Bragg peak, $\lambda_{B}$, undergoes linear fractional shifts as strain, $\varepsilon$, and temperature changes, $\Delta T$, are applied to the FBG [32]:

$\frac{\Delta \lambda}{\lambda}=K_{\varepsilon} \varepsilon+K_{T} \Delta T$.

Here $K_{\varepsilon} \approx 0.8 \mathrm{ppm} / \mu \varepsilon$ and $K_{T} \approx 6.5 \mathrm{ppm} /{ }^{\circ} \mathrm{C}$ are the strain and temperature sensitivity of the FBG, respectively. The diameter of FBG sensors is around $125 \mu \mathrm{m}$. This small physical size is key in this work, as it prevents the sensors from perturbing the strain fields of the components they are monitoring and allows the FBGs to be attached alongside the SAW sensors.

\subsection{Temperature compensation}

It is clear from equations (5) and (7), that SAW and FBG sensors are sensitive to both strain and temperature changes. To monitor strains independently, these effects can be decoupled by monitoring local temperatures using a second, adjacent sensor which is isolated from strain. As ST-cut quartz SAW devices are inherently temperature stable at room temperature [33], it could be argued that temperature compensation is less crucial for shortterm measurements in ambient conditions. However, if SAW sensors are bonded to another component, then frequency shifts may be enhanced by the additional thermal expansion of this component, as demonstrated in Section 3.2.

\subsection{Measurement resolution and range}

Measurement resolutions are limited by how well the interrogation system can resolve shifts in the resonant frequency of the SAW device or the wavelength of the FBG's Bragg peak. In this work, prior to any bonding, SAW sensor strain and temperature resolutions were $3 \mu \varepsilon$ and $6{ }^{\circ} \mathrm{C}$ respectively. FBG strain and temperature resolutions were $1 \mu \varepsilon$ and $0.1^{\circ} \mathrm{C}$ respectively.

Both SAW and FBG sensors are able to monitor between $-20{ }^{\circ} \mathrm{C}$ and $+80^{\circ} \mathrm{C}$, which is an adequate temperature range for most civil engineering applications. While FBG sensors can monitor strains as high as $3 m \varepsilon[34]$, SAW sensor strain ranges are limited by the strength of the quartz substrate to $0.5-1 m \varepsilon$ with more polished substrates demonstrating higher strengths [19]. For high strain monitoring applications, strength limitations can be overcome through sensor packaging design, as described in Section 3.3.

\section{Sensor design and characterisation}

In this work, the SAW sensor's packaging was designed to compensate for the mechanical limitations of the device to allow it to wirelessly monitor large surface displacements in a concrete structure. In this section, the framework for packaging design is outlined, along with computational and experimental methods of verifying the characterisation.

\subsection{Fabrication and interrogation}

The packaged and installed sensor is shown in Figure 2. As shown, the SAW sensor is epoxied to the centre of an $L_{s}=30 \mathrm{~cm}$ long, $b=11.5 \mathrm{~mm}$ wide and $d=0.65 \mathrm{~mm}$ thick carbon-steel shim. Two holes at either end of the shim allow $4 \mathrm{~mm}$ diameter bolts ( $25 \mathrm{~mm}$ long) to be inserted and joined to the shim via induction brazing. The bolts are then grouted into $6 \mathrm{~mm}$ diameter holes, drilled into the surface of a notched concrete beam (the notch was used to encourage cracking at the centre of the beam). Mounting the sensor at a depth of $25 \mathrm{~mm}$ ensures that strain can be transferred from the beam to the sensor even when the surface of the concrete has become spalled or cracked. An FBG sensor was epoxied adjacent to the SAW sensor to verify its strain measurements.

SAW sensors were wirelessly interrogated using an RFID antenna from distances of 1-2 metres at a rate of $1 \mathrm{~Hz}$. The SAW strain sensor had a base resonant frequency (at zero strain and $20^{\circ} \mathrm{C}$ ) of $f_{0}=434.5 \mathrm{MHz}$. The FBG sensor, which had a base wavelength of $\lambda_{B}$ $=1550 \mathrm{~nm}$, was monitored via a fibre connection and using a commercial optical interrogator at a rate of $2 \mathrm{~Hz}$.

\subsection{Temperature characterisation}

Prior to installation onto the concrete beam, the packaged sensor was placed into an environmental chamber, along with an unbonded FBG sensor and an unbonded SAW sensor. The chamber was heated from $0^{\circ} \mathrm{C}$ to $40^{\circ} \mathrm{C}$ in $5{ }^{\circ} \mathrm{C}$ steps. The temperature was held for 2 hours at each step to allow the chamber to reach stable thermal equilibrium.

Figure 3 shows the response of each sensor as a function of the temperature shift from $0^{\circ} \mathrm{C}$ (measured using a local thermocouple). After epoxying to the shim, the SAW sensor's temperature response is only weakly non-linear, due to the large influence of the linear thermal expansion of the shim. Indeed, the enhancement to the FBG's temperature sensitivity after epoxying $\left(\Delta k_{T} \approx 10 \mathrm{ppm} /{ }^{\circ} \mathrm{C}\right)$ confirms this. The influence of bonding to steel is not as pronounced in the SAW sensor because the strain transfer from the shim to the quartz substrate is low, as discussed in Section 


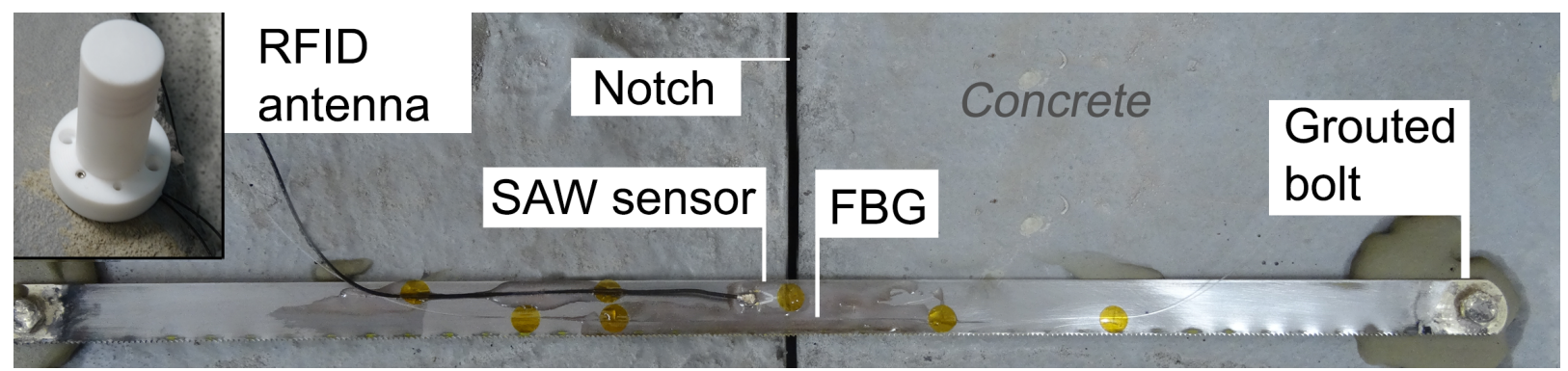

Figure 2. The bottom (tensile surface during bending) face of the notched concrete test beam, showing the sensor packaging (which is comprised of a carbon-steel shim and two steel bolts), grouted into the beam. The wireless SAW sensor, RFID antenna and the FBG used for measurement verification are labelled.
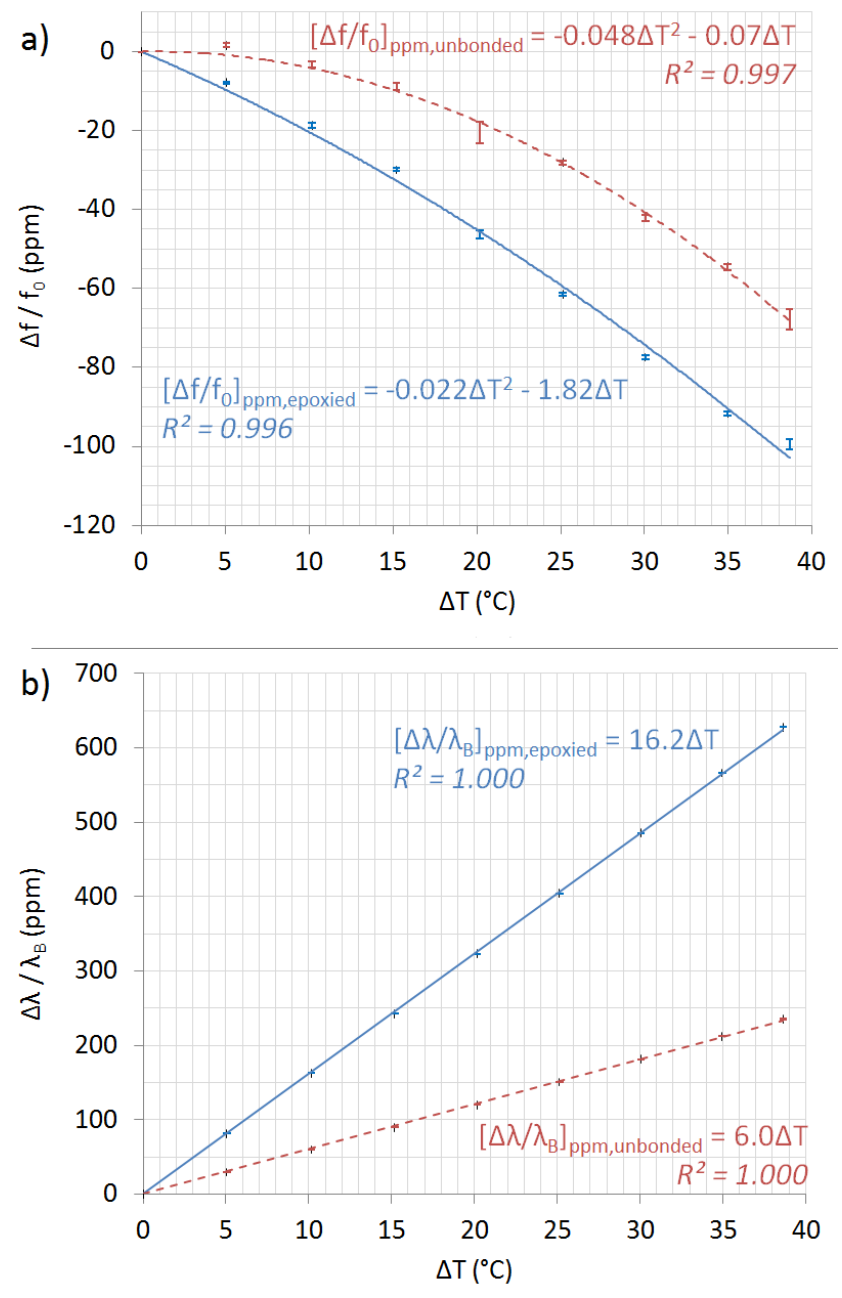

Figure 3. Temperature dependence of a) SAW sensor frequency and b) FBG sensor wavelength, both before and after epoxying to the steel shim. Fractional temperature shifts are measured relative to a datum of $\mathrm{T}=0{ }^{\circ} \mathrm{C}$.

3.3.1. Nevertheless, the added expansion of the steel improves the SAW sensor's temperature resolution to $2{ }^{\circ} \mathrm{C}$.

\subsection{Strain characterisation}

To characterise strain sensitivity prior to installation onto the concrete beam, the bolts of the sensor packaging were clamped and masses of up to $M=$ $0.3 \mathrm{~kg}$ were hung from the centre of the shim to induce three-point bending. In this set up, the maximum strain in the shim is:

$\varepsilon_{\max }=\left(\frac{M g L_{s}}{4}\right)\left(\frac{d}{I_{M} E_{s t}}\right)$,

where $g=9.81 \mathrm{~m} / \mathrm{s}^{2}$ is graviational acceleration, $I_{M}=$ $b d^{3} / 12$ is the beam's second moment of area and $E_{s t} \approx$ $200 \mathrm{GPa}$ is its elasticity.

In practice, empirical measurements of $I_{M}$ and $E_{s t}$ are challenging, as they require accurate knowledge of the shim's geometry and stiffness. However, $I_{M} E_{s t}$ can also be derived from the shim's resonant frequency, $\omega_{c}$, when it is allowed to oscillate as a fixed-free cantilever: $I_{M} E_{s t}=\frac{\omega_{c}^{2} L_{s}^{3}}{3}\left(0.236 M_{\text {shim }}+M_{\text {bolt }}\right) \approx(51 \pm 1) \times 10^{-3} \cdot(9)$ Here $M_{\text {shim }}=17.9 \mathrm{~g}$ and $M_{\text {bolt }}=2.7 \mathrm{~g}$ are the masses of the shim and bolt respectively. To find $\omega_{c}=28.53 \mathrm{rad} / \mathrm{s}$, a high-speed interrogator was briefly used to monitor the frequency of strain oscillations in the epoxied FBG at a rate of $3 \mathrm{kHz}$.

For the three-point bending test, Figure 4 shows the strains measured by the two sensors (derived using equations (5) and (7)), plotted as a function of the applied shim strain, $\varepsilon_{\max }$. Both sensors respond linearly, but the SAW sensor's strain transfer was only $33 \%$ of that of the FBG sensor. Furthermore, the strain transfer to the FBG itself was surprisingly low, as epoxied FBG sensors typically show strain transfers of 0.9 or above [35].

3.3.1. FEM investigation To investigate the strain transfer issue further, a finite element model (FEM) of the sensor packaging under bending was constructed. As shown in Figure 5, during load case 1, the shim was modelled independently, while in load case 2, a quartz SAW sensor was perfectly-bonded to the shim.

Figure 6 shows the axial strain, normalised by $\varepsilon_{\max }$, as a function of model thickness, $y$. In loadcase 1, the FEM's maximum strain is $2.4 m \varepsilon$, which is within $\pm 5 \%$ agreement with equation (8). This demonstrates the validity of the 


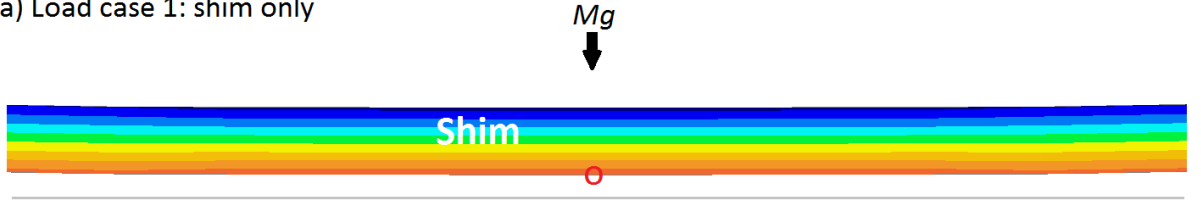

b) Load case 2: shim and SAW

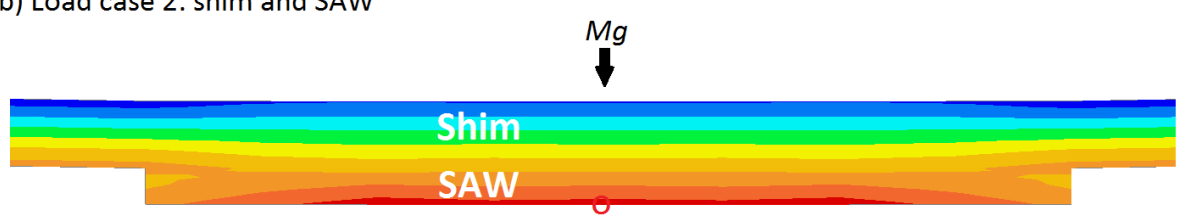

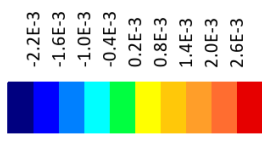

Strain (x-component)

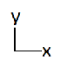

o: max strain location

Figure 5. FEM of the axial strain, $\varepsilon_{x}$, for a) load case 1: the shim only and b) load case 2: the shim bonded to the SAW sensor, under a three-point bending force of $M g=5 \mathrm{~N}$. Only the region near the centre of the shim is shown, and the location of maximum strain in the model circled for each case.

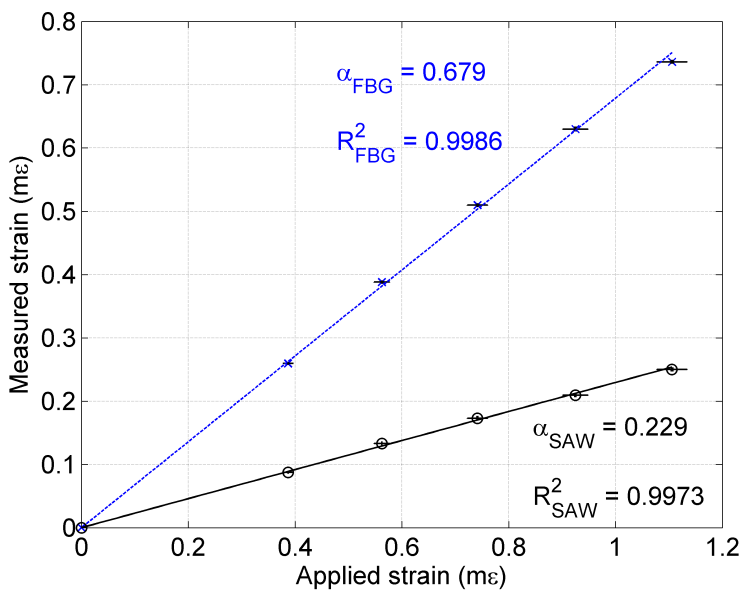

Figure 4. The strain measured by the FBG and SAW sensors as a function of the bending strain applied to the steel shim housing. The strain transfer coefficients and goodness of fit $\left(R^{2}\right.$ values $)$ are also shown. Due to fluctuations in ambient temperature, average measurement errors for the SAW sensor were $1.5 \%$, while those for the FBG sensor were $0.5 \%$.

FEM. When the SAW sensor is added to the model in load case 2, the maximum strain at the surface of the shim decreases by around $50 \%$ (and the results suggest that this decrease acts over the entire width of the shim). The result confirms that the FBG's strain has been diminished in Figure 4 because the SAW sensor's finite thickness has contributed to a higher stiffness, causing it to interfere with the shim's strain field. The drop in strain is, however, more severe in the FEM because perfect bonding between the SAW and the shim is assumed.

Surface acoustic waves are affected by the bulk properties of the quartz substrate, and Figure 5 highlights that the SAW sensor is subjected to a distribution of strains across its thickness. The strain field over the SAW sensor's thickness varies from $0.5 \varepsilon_{\max }$, to $1.1 \varepsilon_{\max }$. This partly explains the lower measured strains of the SAW sensor in Figure 4. In this work, the SAW sensor was bonded from

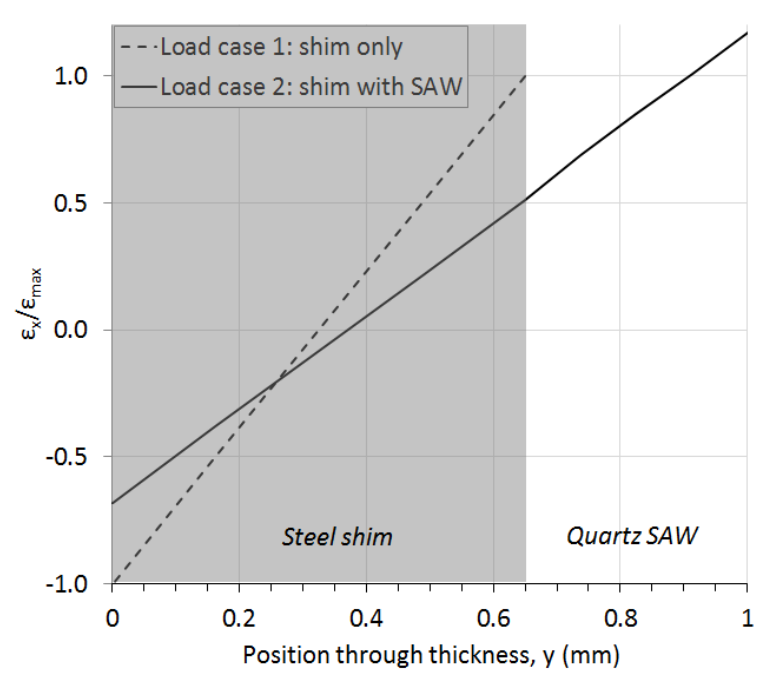

Figure 6. Normalised strain plotted as a function of thickness, $y$ through the FEM. The strains are plotted for loadcase a) shim only, and b) shim with SAW, which are defined in Figure 5. The boundaries of the steel shim are shown by the shaded region on the graph.

one side to the shim, but fully encapsulating the sensors in epoxy may enhance the strain transfer. Misalignments or cracking in the epoxy layer during initial characterisation may also have further reduced strain transfer.

3.3.2. Strain transfer after installation After attachement of the packaged sensor to the concrete beam, the strain transferred to the sensors is further reduced. As the shim is $L_{s}=30 \mathrm{~cm}$ long, its strain after grouting to the concrete beam is described by:

$\varepsilon_{s}=\frac{\Delta L_{s}}{L_{s}}=\frac{u_{B}-u_{A}}{x_{B}-x_{A}}=\frac{1}{L_{s}} \int_{x_{A}}^{x_{B}} \varepsilon(x) d x+\frac{1}{L_{s}} \sum_{j} \Delta w_{j} .(10)$

Here $u_{A, B}$ are the displacements of the anchoring bolts, which are grouted into the concrete beam at locations $x_{A, B}$. The quantities $\Delta w_{j}$ are a set of added 


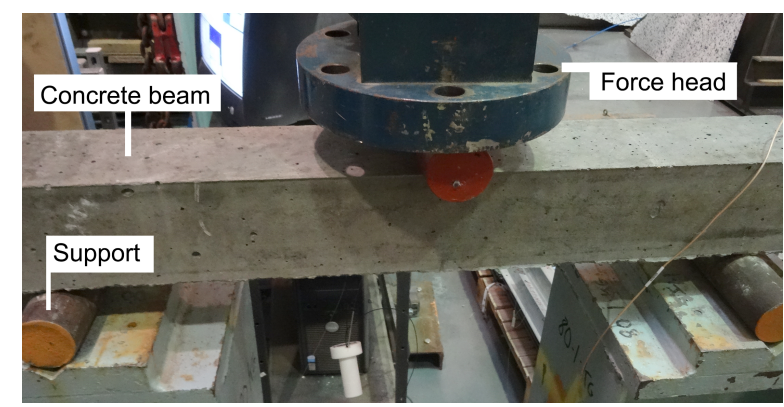

Figure 7. The reinforced concrete beam under three-point bending, showing the supports and the force head.

strain discontinuities caused by the concrete cracking [21]. The integral in equation (10) shows that the sensor housing provides an average measurement of the concrete strain between its two bonding points, resulting in a reduced strain and spatial resolution. Long-gauge sensing is, however, required in this case because concrete is an inhomogenous composite material. Monitoring over small length scales may provide measurements of single crack growth, but longgauge sensors allow multiple cracks and bulk properties to be measured.

As the gauge length is approximately half of the length of the concrete beam, the strain transfer should be reduced by approximately half during three-point bending (ignoring any non-linear effects). The overall strain transfer from the concrete beam to the SAW sensor should therefore be $\alpha_{S A W} / 2 \approx 0.1$. This means that the $3.5 \mathrm{~m} \varepsilon$ sensor strain limit derived in Section 2.1 should not breach the $500 \mu \varepsilon$ strain limit of the SAW sensor during beam testing. It does, however, also mean that the packaged SAW sensor's strain resolution is reduced from $3 \mu \varepsilon$ to $30 \mu \varepsilon$. This is equivalent to reducing the displacement resolution from $1 \mu \mathrm{m}$ to $10 \mu \mathrm{m}$.

\section{Application and results}

The instrumented concrete beam was placed into a three-point bend testing rig, with supports which were $60 \mathrm{~cm}$ apart, as shown in Figure 7. Integrated transducers monitored the stroke (displacement) and applied load of a force head used to deflect the beam. In the first set of experiments, time-varying strokes of frequency $0.05 \mathrm{~Hz}$ and amplitude 0.1, 0.25, 0.5, 0.75 and $1 \mathrm{~mm}$ were applied to the concrete beam. In the second experiment, the load was ramped linearly at a rate of $0.05 \mathrm{kN} / \mathrm{s}$ up until the first signs of beam failure. The load was then held for 90 seconds, before the test was continued at a constant stroke rate of $0.05 \mathrm{~mm} / \mathrm{s}$. This ensured that the beam underwent gradual failure, to capture as much information as possible.

Throughout all tests, a local, unbonded FBG

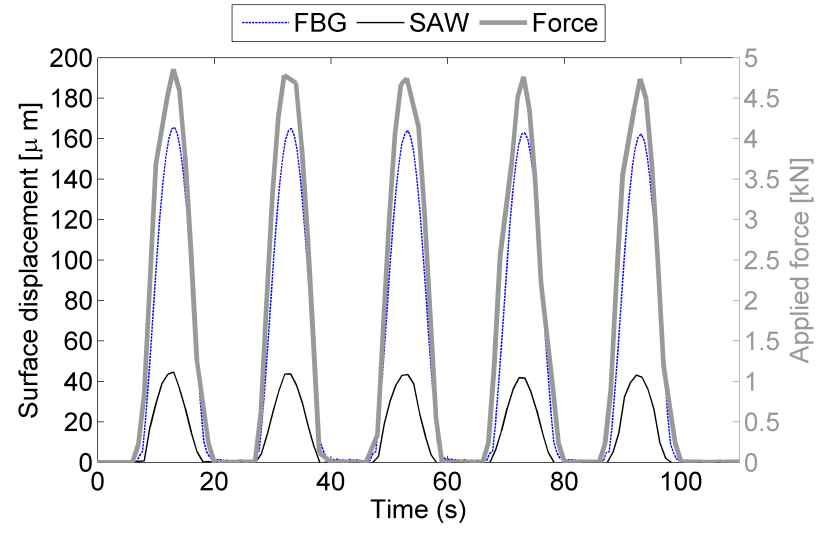

Figure 8. The surface displacements measured by the FBG and SAW sensors during a $4 \mathrm{~mm}$ stroke (approximately $5 \mathrm{kN}$ force) cyclic bending test.

was used as a strain-isolated reference for both SAW sensor and FBG strain sensor measurements, allowing temperature effects to be decoupled.

\subsection{Cyclic loading}

An example of the sensor responses during a $4 \mathrm{~mm}$ cyclic stroke displacement are shown in Figure 8. Although the SAW sensor has a reduced response, it is still able to accurately resolve the time-varying surface displacements in the tensioned surface of the beam.

The peak surface displacement measured during all cyclic tests is plotted against the peak applied force in Figure 9. Non-linear displacement behaviour due to cracking of the beam can clearly be seen. The strain transfer to the SAW sensor has decreased slightly as it now measures strains which are $28 \%$ of the strain in the FBG (rather than the $33 \%$ found in Section 3.3). This reduction is possibly due to microcracking, ageing and relaxation of the epoxy layer used to attach the SAW sensor, as its reasonably large size can cause high stresses at the packaging interface.

\subsection{Linear force ramp to failure}

A linear force ramp was applied to the concrete beam at a rate of $0.05 \mathrm{kN} / \mathrm{s}$ up until the first signs of beam failure at $9 \mathrm{kN}$. The load was then held at $9 \mathrm{kN}$ for 90 seconds, before the force head was controlled at a stroke rate of $0.05 \mathrm{~mm} / \mathrm{s}$ up until complete beam failure. The applied force as a function of the stroke is shown in Figure 10. After the apparatus has resolved initial contact forces, the beam transitions through a linear region. This linear regions lasts up until $5 \mathrm{kN}$, as the prior cyclic loading has already induced cracking up to this level. The response is therefore apparently elastic as the force is taken up by the rebars. Beyond $5 \mathrm{kN}$, the beam begins to behave non-linearly, before 


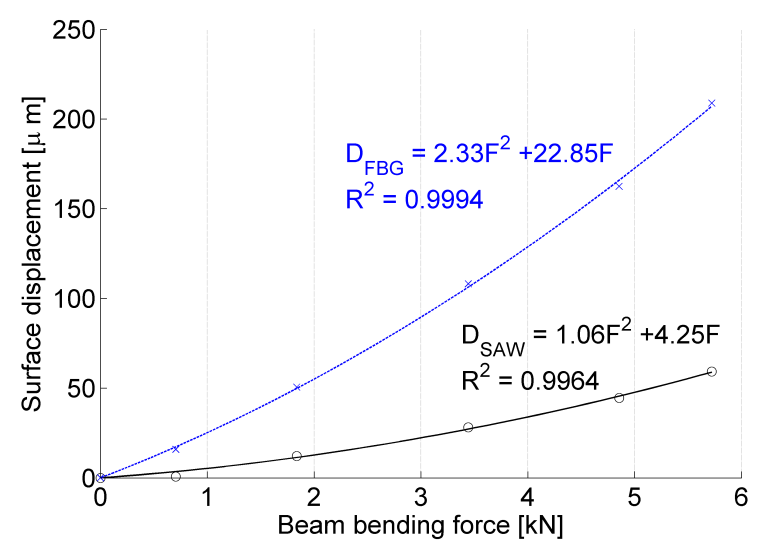

Figure 9. The surface displacements by the FBG and SAW sensors as a function of the cyclic force applied to the concrete beam. The second-order dependence of the displacement on the force arises due to concrete's non-linear behaviour.

failing at a force of $12.6 \mathrm{kN}$. This value broadly agrees with the theoretical failure force of $12 \mathrm{kN}$ derived in Section 2.1.

The SAW sensor response, FBG sensor response and applied force during this test are shown as a time series in Figure 11. The scattered points, obtained by interpolating from Figure 9, are also shown for reference. The agreement between the cyclic and linear ramp tests demonstrates sensor repeatability. However, it is clear that the response during the force ramp test is more linear as the pre-cracked concrete is contributing less to the beam's stiffness, so the surface displacements under bending forces are larger.

Beyond an applied force of $7 \mathrm{kN}$, both sensors measure a reduced surface displacement, even though the stroke rate in Figure 10 is increasing. This is because, at higher loads, the anchorages at the location of the grouted bolts become a defect for crack propagation. This reduces the strain applied to the sensor packaging. At $9 \mathrm{kN}$ (180 seconds into the test) the beam showed significant cracking, and so the force was held. Both the SAW and FBG sensors remained stable during the time period. The beam was finally taken to failure in stroke mode. The sensors show some increase in measured surface displacement before the cracking through the anchorages begins to reverse the strains in the packaging. Note that, throughout the test, the SAW sensor did not exceed its $500 \mu \varepsilon$ limit and so remained operational.

\section{Discussion}

In this work, we demonstrate that sensor packaging can be used to overcome the mechanical limitations of SAW sensors to allow them to provide wireless strain measurements of reinforced concrete, even up until its

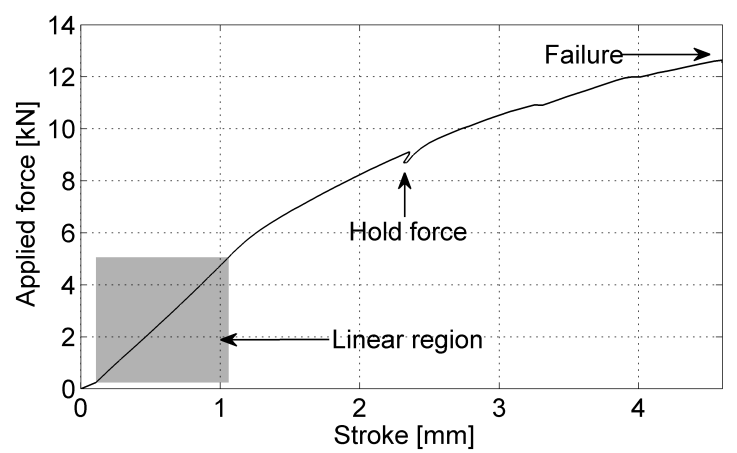

Figure 10. Applied force as a function of stroke (force head displacement) during the linear ramp to failure. The region of linear behaviour is highlighted, along with the force at which the beam was held for 90 seconds, and the point of beam failure.

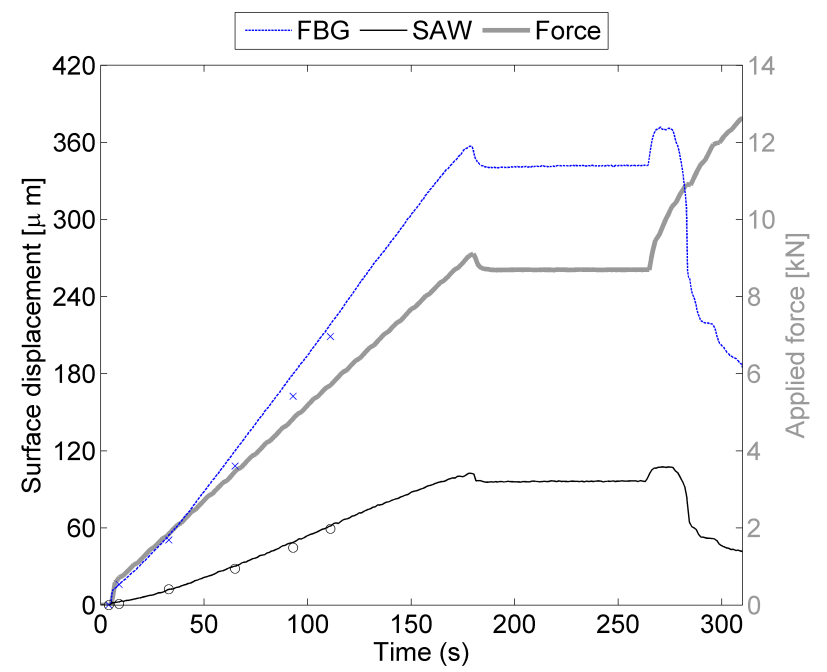

Figure 11. The force and measured surface displacements during the linear force ramp. At 180 seconds, the force was held constant for 90 seconds to demonstrate sensor stability. Beyond this the beam was deflected in constant stroke mode. The scatter points shown are derived from Figure 10, and demonstrate that the sensor behaviour is quite repeatable.

failure. By tuning the length of the packaging, we were able to retrofit sensors to allow the bulk strains of a reinforced concrete beam to be monitored. The sensor could be conveniently characterised and verified prior to installation, so its behaviour after installation was understood.

While details are not reported here, several other methods of surface-mounting SAW sensors onto existing reinforced concrete members were explored in this work. During these feasibility studies, the metrics used to gauge the suitability of each method were:

- Installation - procedures for installation should be as rapid and convenient as possible and suitable for varying weather conditions. 
- Invasiveness - sensor anchorages should not penetrate any deeper than typical concrete covers over rebars. Any drilled anchorages should be of a small diameter.

- Reliability — sensors should be robust and maintain an adequate strain transfer and range.

Epoxy-bonding the SAW sensors to the concrete surface was an obvious and minimally invasive solution. However, as this does not extend the gauge length of the sensors, it only allows for reliable monitoring over a $10 \mathrm{~mm}$ length of a pristine concrete surface. Achieving a reliable bond also required a fully cured primer layer of epoxy, prior to a secondary layer of epoxy to bond the sensor. The method was therefore not only time consuming, but also unsuitable for cold and wet weather conditions. The grouted anchorage method, described in this paper, is more invasive than epoxying, but is suitable for structures with at least $30 \mathrm{~mm}$ of concrete cover (which is the minimum recommended by most building standards [23]). The use of an accelerated grout also makes the method suitable for rapid installation in wet and cold conditions. At room temperatures, the grout was set within minutes and could be fully loaded within a few days. While the bolts did provide the point of failure in the beam tests, this was due to the large size of the holes relative to the beam thickness and applied loads, and may not pose an issue for larger structure.

SAW sensors naturally lend themselves to multiplexing. The initial frequency of SAW devices can be customised during fabrication so that frequencydivision-multiplexed arrays of SAW devices can be used to monitor quasi-distributed strains, displacements and temperatures in large structures with a single interrogation device. Even more sensors can be interrogated with a single interrogator if time and codedivision multiplexing are used [36]. The advantage of wireless sensing over FBG technology is that the physical complexity of the interrogation network is reduced: considerations about where to trail fibres or wires are removed. However, as with any wireless device, achieving an efficient coupling between the transmitting and receiving antennas is essential. As SAW technology utilises RFID, the wireless interrogation distance is typically limited to a few metres in air, but signals are dramatically attenuated by concrete, soil or metal. As such, SAW devices are not suitable for monitoring concrete assets below ground, or those in steel-congested areas.

While the strain/displacement measurement range was increased in this work, the trade-off was a reduction in measurement sensitivity. If the application demands a higher resolution, then this can be facilitated by reducing the thickness of the SAW device or by reducing the gauge length of the sensor.
In this work, thermal and strain effects were assumed to act independently, and this is not an atypical approach for sensor characterisation. However, as SAW sensors operate based on changes on both phase velocity and pitch, strain sensitivity will likely have some dependence on operating temperature, and viceversa. In an environment with large fluctuations in both strain and temperature, these effects should be accounted for [37].

\section{Conclusions}

We have demonstrated that wireless surface acoustic wave sensors can be used for monitoring large (2$4 m \varepsilon$ ) strains and crack opening of up to $1 \mathrm{~mm}$ during cyclic and static loading of reinforced concrete structures. The strain accuracy of the SAW sensor measurements was verified using computational models and a secondary fibre-based strain sensor. By careful design of the packaging, the strain transfer from the concrete beam to the SAW sensor could be reduced to around 10-20\% to prevent the SAW device's quartz substrate from reaching its fracture limit during large structural deflections. The technology may now be suitable for retrofitting to existing concrete structures to wirelessly monitor surface displacements and crack opening.

\section{References}

[1] Spencer B F, Ruiz-Sandoval M E and Kurata N Struct. Control Health Monit. 11 349-368

[2] Lynch J P and Loh K J Shock Vib. Dig. 38 91-128

[3] Cho S, Spencer B F, Jo H, Li J and Kim R E Bridge monitoring using wireless smart sensors SPIE Newsroom

[4] Kotowsky M, Dowding C and Fuller K Wireless sensor networks to monitor crack growth on bridges Developing a Research Agenda for Transportation Infrastructure Preservation and Renewal

[5] Lynch J P Philos. Trans. R. Soc. A 365 345-372

[6] Popovici E, Magno M and Marinkovic S Power management techniques for wireless sensor networks: A review 5th International Workshop on Advances in Sensors and Interfaces pp 194-198

[7] White R New prospects for acoustic sensors: an overview 41st Annual Symposium on Frequency Control. 1987 pp 333-338

[8] Varadan V K and Varadan V V Smart Mater. Struct. 9953

[9] Kalinin V Passive wireless strain and temperature sensors based on SAW devices IEEE Radio and Wireless Conference pp 187-190

[10] Drafts B IEEE Trans. Microw. Theory Tech. 49 795-802

[11] Wolff U, Dickert F L, Fischerauer G K, Greibl W and Ruppel C C W IEEE Sens. J. 1 4-13

[12] Buff W SAW sensors for direct and remote measurement IEEE Ultrasonics Symposium vol 1 pp 435-443

[13] Glisic B and Inaudi D Crack monitoring in concrete elements using long-gage fiber optic sensors

[14] Kalansuriya P, Bhattacharyya R and Sarma S IEEE Sens. J. 13 1564-1570

[15] Sahay P, Kaya M and Wang C 13 39-57 
[16] Kim J S, Vinoy K J and Varadan V K Wireless health monitoring of cracks in structures with MEMS-IDT sensors SPIE's 9th Annual International Symposium on Smart Structures and Materials vol 4700 pp 342-353

[17] Li Z, Li F, Zdunek E, Landis E and Shah S ACI Mater. J. 95

[18] Zhang X, Wang F, Wang Z, Li W and He D Intelligent tires based on wireless passive surface acoustic wave sensors 7 th International IEEE Conference on Intelligent Transportation Systems pp 960-964

[19] Chao H and Parker T Tensile fracture strength of ST cut quartz 37th Annual Symposium on Frequency Control. 1983 pp $116-124$

[20] Chang P C, Flatau A and Liu S C Structural Health Monitoring 2 257-267

[21] Glisic B Meas. Sci. Technol. 22035206

[22] Hwang L and Rizkalla S Effective tensile stress-strain characteristics for reinforced concrete Proceedings of the Canadian Society of Civil Engineering pp 129-147

[23] American Concrete Institute Building code requirements for structural concrete (ACI 318), ACI committee 318

[24] Whitney C S ACI J. Proc. 33

[25] Shiokawa S and Kondoh J Jpn. J. Appl. Phys. 432799

[26] Springer A, Weigel R, Pohl A and Seifert F 9 745-756

[27] Levien L, Prewitt C and Weidner J Am. Mineral. 65 920930

[28] Moller F and Kuhn J Sensors and Actuators A: Physical $3073-75$

[29] Ballantine D S, White R M, Martin S J, Ricco A J, Zellers E T, Frye G C and Wohltjen H Acoustic Wave Sensors: Theory, Design, \& Physico-Chemical Applications (Academic Press) ISBN 978-0-08-052333-0

[30] Majumder M, Gangopadhyay T K, Chakraborty A K, Dasgupta K and Bhattacharya D K Sensors and Actuators A: Physical 147 150-164

[31] Leung C K Y, Wan K T, Inaudi D, Bao X, Habel W, Zhou $\mathrm{Z}$, Ou J, Ghandehari M, Wu H C and Imai M Mater Struct 48 871-906

[32] Hill K O and Meltz G J. Light. Technol. 15 1263-1276

[33] Yamazaki T, Iizawa K, Kanna S and Takagi M Jpn. J. Appl. Phys. 423136

[34] Yoon H J and Kim C G Smart Material Structures 16 13151319

[35] Wan K T, Leung C K Y and Olson N G Smart Mater. Struct. 17035037

[36] Malocha D C, Gallagher M, Fisher B, Humphries J, Gallagher D and Kozlovski N 13 5897-5922

[37] Alzuaga S, Michoulier E, Friedt J M, Martin G, Berthelot $\mathrm{P}$, Ptrini $\mathrm{V}$ and Ballandras $\mathrm{S}$ Characterization of the thermal dependence of SAW stress sensitivity 10me Congrs Franais d'Acoustique 